\title{
LOCALLY UNIVALENT FUNCTIONS, VMOA AND THE DIRICHLET SPACE
}

\author{
EVA A. GALLARDO-GUTIÉRREZ, MARÍA J. GONZÁLEZ, FERNANDO \\ PÉREZ-GONZÁLEZ, CHRISTIAN POMMERENKE AND JOUNI RÄTTYÄ
}

\begin{abstract}
We study geometric properties of the image of the unit circle under a bounded locally univalent function $g$ such that $\log g^{\prime}$ belongs either to the Dirichlet space $\mathcal{D}, \mathrm{VMOA}$ or the little Bloch space $\mathcal{B}_{0}$. Concerning VMOA and $\mathcal{B}_{0}$, our findings generalize the corresponding results for conformal maps shown by Pommerenke in the late seventies. In the case of $\mathcal{D}$, we give a strictly geometric necessary condition for $g$ to satisfy $\log g^{\prime} \in \mathcal{D}$, and also offer two different "semi-geometric" characterizations of when $\log g^{\prime} \in \mathcal{D}$.
\end{abstract}

\section{INTRODUCTION AND RESULTS}

Let $\mathcal{C}$ be a (closed) Jordan curve in the complex plane $\mathbb{C}$ and let $C\left(w_{l}, w_{2}\right)$ denote the smaller arc of $\mathcal{C}$ between the points $w_{1}$ and $w_{2}$ on $\mathcal{C}$. Recall that $\mathcal{C}$ is called asymptotically conformal if

$$
\max _{w \in \mathcal{C}\left(w_{1}, w_{2}\right)} \frac{\left|w_{2}-w\right|+\left|w-w_{1}\right|}{\left|w_{2}-w_{1}\right|} \rightarrow 1, \quad \text { as } \quad\left|w_{2}-w_{1}\right| \rightarrow 0,
$$

and quasi-conformal if this maximum is uniformly bounded for all $w_{1}, w_{2} \in \mathcal{C}$. The latter case occurs if and only if $\mathcal{C}$ is the image of a circle under a quasiconformal mapping of $\mathbb{C}$ [22, Theorem 9.14], and therefore quasi-conformal curves are usually called quasi-circles. The concept of asymptotically conformal curves was introduced by Becker [2] in the early seventies, and it fits nicely between the theories of quasi-conformal and smooth curves.

2000 Mathematics Subject Classification. Primary 30C45; Secondary 30C55.

Key words and phrases. (little) Bloch space, VMOA, Dirichlet space, conformal map, locally univalent function, asymptotically conformal curve, asymptotically smooth curve.

The first author is partially supported by Plan Nacional I+D grant no. MTM2007-61446 and Gobierno de Aragón research group Análisis Matemático y Aplicaciones, ref. DGA E-64. The second author is partially supported by Plan Nacional I+D grant no. MTM2008-00145 and grant PIV2008. The third and fifth authors are partially supported by Plan Nacional I $+\mathrm{D}$ grant no. MTM2007-30907-E, MTM2008-05891, and by the ESF PESC Research Network HCAA. The fourth author is partially supported by Plan Nacional I+D grant no. MTM2006-14449C02-01. The fifth author is also partially supported by the Emil Aaltonen Foundation and the Academy of Finland grant no. 121281. Finally, the second author is very grateful to the Centre de Recerca Matemática for the invitation to participate in a special research programme in Analysis, held in the spring of 2009. This paper was completed on that occasion. 
In addition, recall that if $\mathcal{C}$ is a rectifiable Jordan curve and $l\left(w_{1}, w_{2}\right)$ denotes the length of the shorter arc on $\mathcal{C}$ joining $w_{1}$ and $w_{2}$, then $\mathcal{C}$ is said to be asymptotically smooth if

$$
\frac{l\left(w_{1}, w_{2}\right)}{\left|w_{2}-w_{1}\right|} \rightarrow 1, \quad \text { as } \quad\left|w_{2}-w_{1}\right| \rightarrow 0,
$$

and quasi smooth if this quotient is uniformly bounded for all $w_{1}, w_{2} \in \mathcal{C}$. Inner domains of quasi smooth curves are also known as chord-arc or Lavrentiev domains [19].

Denoting by $g$ a conformal map taking $\mathbb{D}$ onto the inner domain of a Jordan curve $\mathcal{C}$, a fundamental question in the theory of conformal maps is the relationship between the geometric properties of $\mathcal{C}$ and the analytic properties of $g$. In this spirit, in 1978, Pommerenke [20] characterized both asymptotically conformal and asymptotically smooth curves in terms of analytic properties of $\log g^{\prime}$. More precisely, he showed that $\mathcal{C}$ is asymptotically conformal (resp. asymptotically smooth) if and only if $\log g^{\prime}$ belongs to the little Bloch space $\mathcal{B}_{0}$ (resp. VMOA). Further, concerning asymptotically conformal curves, Pommerenke and Warschawski [23] showed in 1982 that the geometric quantity

$$
\eta(\delta)=\sup _{\left|w_{1}-w_{2}\right|<\delta} \sup _{w \in \mathcal{C}\left(w_{1}, w_{2}\right)}\left(\frac{\left|w_{2}-w\right|+\left|w-w_{1}\right|}{\left|w_{2}-w_{1}\right|}-1\right)^{1 / 2}
$$

and the analytic quantity

$$
\beta(\delta)=\sup _{1-\delta \leq|\zeta|<1}(1-|\zeta|)\left|\frac{g^{\prime \prime}(\zeta)}{g^{\prime}(\zeta)}\right|
$$

are of (almost) the same order of magnitude as $\delta \rightarrow 0^{+}$. See also the related results by Rodin and Warschawski [25].

More recently, in 1991, Astala and Zinsmeister [1] studied the set of conformal maps $g$ such that $\log g^{\prime}$ belongs to BMOA. In particular, they showed that $\log g^{\prime} \in \mathrm{BMOA}$ if and only if the measure $\left|S_{g}(z)\right|^{2}\left(1-|z|^{2}\right)^{3} d A(z)$, induced by the Schwarzian derivative $S_{g}$ of $g$, is a Carleson measure on $\mathbb{D}$. In 1994 Bishop and Jones [5] obtained a complete (analytic and geometric) description of those simply connected domains $\Omega$ such that any Riemann map $g$ of $\mathbb{D}$ onto $\Omega$ satisfies $\log g^{\prime} \in \mathrm{BMOA}$, see also [13, Chapters VII and X]. One of the geometric characterizations of these BMOA-domains immediately gives their bi-Lipschitz invariance, a property which a priori is far from being obvious. For characterizations in terms of the Schwarzian derivative of when $\log g^{\prime}$ belongs either to $\mathcal{D}$, VMOA or $\mathcal{B}_{0}$, we refer to [3] and [18].

The aim of this paper is twofold. On one hand, we are interested in the relationship between the geometric properties of $\mathcal{C}=g(\mathbb{T})$ and the analytic properties of $g$ when $g$ is a locally univalent function on $\mathbb{D}$, continuous on $\overline{\mathbb{D}}$, such that $\mathcal{C}$ is closed but might have self-intersections. The results shown for locally univalent functions generalize those already mentioned for conformal maps and related to 
VMOA and the little Bloch space $\mathcal{B}_{0}$. Roughly speaking, "the same sort" of results hold even when the assumption on $g$ of being a Riemann map is omitted. The techniques we will exploit make an extensive use of an argument of localization for locally univalent maps. This allows us to consider the curve $\mathcal{C}$ as a finite union of Jordan arcs instead of a closed curve with possible self-intersections.

On the other hand, our goal is to provide a geometric characterization of $g(\mathbb{T})$ such that the locally univalent function $g$, continuous on $\overline{\mathbb{D}}$, satisfies $\log g^{\prime} \in \mathcal{D}$. Let us point out that in the case of the Dirichlet space $\mathcal{D}$ no geometric characterizations are known even in the case when $g$ is conformal.

The rest of this introductory section (and the paper) is organized as follows. We will first recall the definitions of the spaces which our results concern. Then we will state our main results along with examples which will shed some light on the results proved. Each of the results will be proved in the subsequent sections along with the tools needed in the proofs.

Preliminaries on function spaces. Let $\mathbb{D}$ denote the unit disc of the complex plane $\mathbb{C}$ and $\mathcal{H}(\mathbb{D})$ the algebra of all analytic functions in $\mathbb{D}$. For $0<p<\infty$, the Hardy space $H^{p}$ consists of those $f \in \mathcal{H}(\mathbb{D})$ for which

$$
\|f\|_{H^{p}}^{p}:=\lim _{r \rightarrow 1^{-}} M_{p}^{p}(r, f):=\lim _{r \rightarrow 1^{-}} \frac{1}{2 \pi} \int_{0}^{2 \pi}\left|f\left(r e^{i t}\right)\right|^{p} d t<\infty .
$$

The space of bounded analytic functions on $\mathbb{D}$ is denoted by $H^{\infty}$. A classical result due to Fatou states that every Hardy function has a radial limit almost everywhere on the unit circle $\mathbb{T}:=\{z:|z|=1\}$, see, for instance, [11]. Throughout this work, $f(\zeta)$ denotes the radial limit of $f$ at $\zeta \in \mathbb{T}$.

The space BMOA of analytic functions with bounded mean oscillation on $\mathbb{T}$ consists of those $f \in H^{2}$ for which

$$
\|f\|_{\mathrm{BMOA}}^{2}:=\sup _{\zeta \in \mathbb{D}}\left\|f_{\zeta}\right\|_{H^{2}}^{2}:=\sup _{\zeta \in \mathbb{D}} \frac{1}{2 \pi} \int_{\mathbb{T}}|f(z)-f(\zeta)|^{2} \frac{1-|\zeta|^{2}}{|z-\zeta|^{2}}|d z|<\infty,
$$

where

$$
f_{\zeta}(z):=\left(f \circ \varphi_{\zeta}\right)(z)-f(\zeta), \text { and } \varphi_{\zeta}(z):=\frac{\zeta-z}{1-\bar{\zeta} z} .
$$

Alternative characterizations of BMOA, as well as a systematic treatment of the subject, can be found in [12, Chapter VI].

The space VMOA consists of those $f \in H^{2}$ for which the integral in (1.1) tends to zero as $\zeta$ approaches to the boundary $\mathbb{T}$. BMOA is a subspace of the Bloch space

$$
\mathcal{B}:=\left\{f \in \mathcal{H}(\mathbb{D}):\|f\|_{\mathcal{B}}:=\sup _{z \in \mathbb{D}}\left|f^{\prime}(z)\right|\left(1-|z|^{2}\right)<\infty\right\},
$$

and VMOA is a subspace of both BMOA and the little Bloch space

$$
\mathcal{B}_{0}:=\left\{f \in \mathcal{H}(\mathbb{D}): \lim _{|z| \rightarrow 1^{-}}\left|f^{\prime}(z)\right|\left(1-|z|^{2}\right)=0\right\} .
$$


Finally, let us recall that $f \in \mathcal{H}(\mathbb{D})$ belongs to the classical Dirichlet space $\mathcal{D}$ if

$$
\|f\|_{\mathcal{D}}^{2}:=\frac{1}{\pi} \int_{\mathbb{D}}\left|f^{\prime}(z)\right|^{2} d A(z)+|f(0)|^{2}<\infty
$$

where $d A(z)$ denotes the element of the Lebesgue area measure on $\mathbb{D}$. It is obvious that $\mathcal{D}$ is contained in VMOA, and hence the spaces of interest in this study satisfy $\mathcal{D} \subset$ VMOA $\subset \mathcal{B}_{0}$. Note also that the integral above corresponds to the area of image of $\mathbb{D}$ under $f$ counting multiplicities. If $f(z)=\sum_{n=0}^{\infty} a_{n} z^{n}$, then Parseval's formula shows that

$$
\|f\|_{\mathcal{D}}^{2}=\sum_{n=1}^{\infty} n\left|a_{n}\right|^{2}+\left|a_{0}\right|^{2} .
$$

A classical result due to Beurling [4] states that if $f$ is a Dirichlet function, then its radial limits exist in $\mathbb{T}$ outside of a possible exceptional set of zero logarithmic capacity, see also [7, p. 55]. Recall that if $E$ is a Borel set in $\mathbb{T}$ and $\Lambda_{E}$ denotes the class of distributions of mass 1 on $E$, i.e., non-negative set functions $\mu$ with total mass 1 and support $S_{\mu}$ contained in $E$, the logarithmic capacity ${ }^{1}$ of $E$ is defined as

$$
\exp \left(-\inf _{\Lambda_{E}}\{I(\mu)\}\right)
$$

where

$$
I(\mu)=\int_{\mathbb{T}} \int_{\mathbb{T}} \log \frac{1}{|\xi-\eta|} d \mu(\xi) d \mu(\eta)
$$

is the logarithmic energy integral of $\mu$. The logarithmic capacity of $E$ will be denoted by $\operatorname{cap}(E)$.

Main results and examples. We proceed to state our main results. The first theorem establishes the possible growth, measured in terms of area integrals or Hardy spaces, of the derivative of a locally univalent function $g$ on $\mathbb{D}$ when $\log g^{\prime}$ belongs either to $\mathcal{B}_{0}, \mathrm{VMOA}$ or $\mathcal{D}$. It is clear that this result could have been stated in terms of functions $f$ and their exponentials $\exp (f)$ instead of $\log g^{\prime}$ and $g^{\prime}$. In fact, this is the notation used while proving these implications in Section 2.

Theorem 1. Let $g \in \mathcal{H}(\mathbb{D})$ be locally univalent. Then the following assertions hold:

(1) If $\log g^{\prime} \in \mathcal{B}_{0}$, then

$$
\int_{\mathbb{D}}\left|g^{\prime}(z)\right|^{p}\left(1-|z|^{2}\right)^{\alpha} d A(z)<\infty
$$

for all $p>0, \alpha>-1$.

(2) If $\log g^{\prime} \in \mathrm{VMOA}$, then $g^{\prime} \in H^{p}$ for all $p>0$.

\footnotetext{
${ }^{1}$ Some authors define the logarithmic capacity of $E$ by $\left(\inf _{\Lambda_{E}}\{I(\mu)\}\right)^{-1}$. In our case, both definitions are consistent because we will deal with sets of logarithmic capacity zero. For more about capacities see [7] and [15].
} 
(3) If $\log g^{\prime} \in \mathcal{D}$, then

$$
\int_{\mathbb{D}}\left|g^{\prime}(z)\right|^{p-2}\left|g^{\prime \prime}(z)\right|^{2}\left(\log \frac{1}{|z|}\right)^{\alpha} d A(z)<\infty
$$

for all $p, \alpha>0$.

If $g$ is locally univalent on $\mathbb{D}$ such that $\log g^{\prime} \in \mathcal{B}_{0}$, then Part (1) in Theorem 1 implies that $M_{\infty}\left(r, g^{\prime}\right):=\max _{|z|=r}\left|g^{\prime}(z)\right|$ can not exceed the growth of $(1-r)^{-\frac{\alpha+2}{p}}$, as $r \rightarrow 1^{-}$, for any $p>0$ and $\alpha>-1$. Choosing $\alpha=0$ and $p>2$, it follows that $g$ must be continuous on $\overline{\mathbb{D}}$. The same conclusion holds under the assumption that $\left\|\log g^{\prime}\right\|_{\mathcal{B}}$ is sufficiently small. More precisely, a reasoning similar to that in Section 2.1 shows that for any given $p>0$ and $\alpha>-1$ there exists $\varepsilon>0$ such that $\left\|\log g^{\prime}\right\|_{\mathcal{B}}<\varepsilon$ implies (1.3).

Part (2) in Theorem 1 is known, see for instance (3.5) in [21], and also [9] for related results. Nevertheless, an alternative proof is included for the sake of completeness. Moreover, a reasoning similar to that in Section 2.2 shows that for a given $p>0$ there exists $\varepsilon>0$ such that $\left\|\log g^{\prime}\right\|_{\mathrm{BMOA}}<\varepsilon$ implies $g^{\prime} \in H^{p}$.

It is also worth noticing that an application of the Chang-Marshall Theorem [8] shows that if $\log g^{\prime} \in \mathcal{D}$, then $g^{\prime} \in H^{p}$ for all $p>0$. Needless to say that Part (3) in Theorem 1 does not follow by such an implication. Indeed, the well-known Hardy-Stein-Spencer identity says that (1.4) with $\alpha=1$ is satisfied if and only if $g^{\prime} \in H^{p}$, see (2.1) in Section 2.2.

Example 1 shows that there exists a locally univalent function $g$ such that $\log g^{\prime} \in \mathcal{B}_{0}$ but whose derivative does not belong to $\cup_{p>0} H^{p}$. This illustrates the sharpness of Parts (1) and (2) in Theorem 1.

Example 1. Let $g$ be a locally univalent function defined by

$$
\log g^{\prime}(z):=\sum_{k=1}^{\infty} a_{k} z^{k^{k}}=\sum_{k=1}^{\infty} k^{-\frac{1}{2}} z^{k^{k}}
$$

Now $\log g^{\prime}$ is a lacunary series, and hence $\log g^{\prime} \in \mathcal{B}_{0}$ since $\lim \sup _{k \rightarrow \infty}\left|a_{k}\right|=0$. Therefore $g$ satisfies (1.3) for all $p>0$ and $\alpha>-1$ by Part (1) in Theorem 1 . On the other hand, $\sum_{k=1}^{\infty}\left|a_{k}\right|^{2}=\sum_{k=1}^{\infty} \frac{1}{k}=\infty$, and hence $\log g^{\prime}$ has angular limits almost nowhere on $\mathbb{T}$ by Zygmund's theorem [27, p. 203]. Therefore $g^{\prime}$ is not of bounded characteristic and thus $g^{\prime}$ does not belong to $\cup_{p>0} H^{p}$.

By Example 1 there exists a locally univalent function $g$ such that $\log g^{\prime} \in \mathcal{B}_{0}$, but $g^{\prime}$ has angular limits almost nowhere on $\mathbb{T}$. If $\log g^{\prime} \in \mathrm{VMOA}$, then $g^{\prime} \in H^{p}$ by Part (2) in Theorem 1 and hence $g^{\prime}$ has radial limits almost everywhere on $\mathbb{T}$ by the Fatou Theorem. The following example shows that $g^{\prime}$ might not have a limit in a dense subset of $\mathbb{T}$ when $\log g^{\prime} \in \mathcal{D}$. 
Example 2. A direct calculation shows that $\log g_{\zeta}^{\prime}(z):=\log \log (3 /(1-\bar{\zeta} z)) \in \mathcal{D}$ for all $\zeta \in \mathbb{T}$. Since $\mathcal{D}$ is linear, it follows that

$$
\log g^{\prime}(z):=\log \prod_{k}\left(\log \frac{3}{1-\bar{\zeta}_{k} z}\right)^{\lambda_{k}}=\sum_{k} \lambda_{k} \log \log \frac{3}{1-\bar{\zeta}_{k} z}=\sum_{k} \lambda_{k} \log g_{\zeta_{k}}^{\prime}
$$

belongs to $\mathcal{D}$ if $\sum_{k}\left|\lambda_{k}\right|<\infty$. If $\left\{\zeta_{k}\right\}$ is dense in $\mathbb{T}$, then $g^{\prime}$ does not have limit in a dense subset of $\mathbb{T}$.

We now turn to consider the points in $g(\mathbb{T})$ where tangents exist. In order to state the results, we recall the definition of an arc length parametrization of a curve.

Definition 1. Let $g$ be a conformal map of $\mathbb{D}$ onto the inner domain of a rectifiable Jordan curve $\mathcal{C}$. Let $l=l(\mathcal{C})$ denote the length of $\mathcal{C}$ and let $\psi:[0, l] \rightarrow \mathbb{T}$ stand for the inverse function of

$$
z \mapsto \int_{0}^{\arg z}\left|g^{\prime}\left(e^{i t}\right)\right| d t, \quad z \in \mathbb{T} .
$$

Then $\omega(s)=g(\psi(s)), 0 \leq s \leq l$, is an arc length parametrization of $\mathcal{C}$ such that $\omega(0)=g(1)$.

Note that the integral in (1.5) is well defined since $\mathcal{C}$ is assumed to be rectifiable and therefore $g^{\prime} \in H^{1}$, see, for instance, [19, Theorem 6.8]. Hence, by the Fatou Theorem, its radial limits exist almost everywhere on $\mathbb{T}$.

Throughout the paper, the notation $\omega(s)$ is also used for the arc length parametrization of $\mathcal{C}=g(\mathbb{T})$ when $g$ is a locally univalent function on $\mathbb{D}$ such that $\mathcal{C}$ is a closed rectifiable curve with self-intersections.

Theorem 2. Let $g \in \mathcal{H}(\mathbb{D})$ be locally univalent. Then the following assertions hold:

(1) If $\log g^{\prime} \in \mathcal{B}_{0}$ and $g^{\prime}(\zeta)=\lim _{r \rightarrow 1^{-}} g^{\prime}(r \zeta) \neq 0, \infty$ exists for some $\zeta \in \mathbb{T}$, then

$$
\frac{g(z)-g(\zeta)}{z-\zeta} \rightarrow g^{\prime}(\zeta), \quad z \rightarrow \zeta, \quad z \in \overline{\mathbb{D}}
$$

(2) If $\log g^{\prime} \in \mathrm{VMOA}, g^{\prime}(\zeta)=\lim _{r \rightarrow 1^{-}} g^{\prime}(r \zeta) \neq 0, \infty$ exists and $\zeta=\psi(\sigma)$, then the arc length parametrization $\omega(s)=g(\psi(s)), 0 \leq s \leq l$, of $\mathcal{C}$ satisfies

$$
\omega^{\prime}(\sigma)=\lim _{s \rightarrow \sigma} \frac{\omega(s)-\omega(\sigma)}{s-\sigma}=i \zeta \frac{g^{\prime}(\zeta)}{\left|g^{\prime}(\zeta)\right|}=e^{i \theta(\sigma)},
$$

where $\theta(\sigma):=\frac{\pi}{2}+\arg \left(\zeta g^{\prime}(\zeta)\right)$. In particular, $\mathcal{C}$ has a tangent at $\omega(\sigma)$ with the angle $\theta(\sigma)$.

(3) If $\log g^{\prime} \in \mathcal{D}$, then the arc length parametrization $\omega(s)=g(\psi(s)), 0 \leq s \leq$ $l$, of $\mathcal{C}$ satisfies (1.6) for all $\sigma \in[0, l] \backslash E$ with $\operatorname{cap}(E)=0$. In particular, $\mathcal{C}$ has a tangent outside of a possible exceptional set of zero logarithmic capacity. 
If $g$ is conformal, then Part (1) in Theorem 2 follows by [20, Corollary 2]. The same conlusion for locally univalent functions is obtained by using the localizing technique to be introduced in Section 3.1. Part (1) along with the other two statements will be proved in Section 4.

In order to state our next result, which is one of the cores of this work, we need to generalize the definitions of asymptotically conformal and asymptotically smooth Jordan curves to self-intersecting ones.

Definition 2. Let $w:[0,2 \pi] \rightarrow \mathcal{C}$ be a parametrization of a closed curve $\mathcal{C}$. Let $\mathcal{C}\left(w\left(t_{1}\right), w\left(t_{2}\right)\right)$ denote the smaller arc on $\mathcal{C}$ connecting $w\left(t_{1}\right)$ and $w\left(t_{2}\right)$, that is, the one with $\left|t_{1}-t_{2}\right|<\pi$. The curve $\mathcal{C}$ is called asymptotically conformal if

$$
\max _{w \in \mathcal{C}\left(w\left(t_{1}\right), w\left(t_{2}\right)\right)} \frac{\left|w\left(t_{2}\right)-w\right|+\left|w-w\left(t_{1}\right)\right|}{\left|w\left(t_{2}\right)-w\left(t_{1}\right)\right|} \rightarrow 1, \quad \text { as } \quad\left|t_{2}-t_{1}\right| \rightarrow 0 .
$$

Moreover, if $\mathcal{C}$ is a rectifiable, then $\mathcal{C}$ is called asymptotically smooth if

$$
\frac{l\left(w\left(t_{1}\right), w\left(t_{2}\right)\right)}{\left|w\left(t_{2}\right)-w\left(t_{1}\right)\right|} \rightarrow 1, \quad \text { as } \quad\left|t_{2}-t_{1}\right| \rightarrow 0 .
$$

If $\mathcal{C}$ is a Jordan curve, then $\left|w\left(t_{2}\right)-w\left(t_{1}\right)\right| \rightarrow 0$ if and only if $\left|t_{2}-t_{1}\right| \rightarrow 0$, and therefore the definitions above are equivalent to the classical ones for Jordan curves, stated at the beginning of the introduction.

With this definition in hand, we are in position to generalize Pommerenke's characterizations of asymptotically conformal and asymptotically smooth Jordan curves (see [20],[21] or the monograph [19]) for closed curves with selfintersections.

Theorem 3. Let $g \in \mathcal{H}(\mathbb{D})$ be locally univalent. Then the following assertions hold:

(1) $\log g^{\prime} \in \mathcal{B}_{0}$ if and only if $g$ is continuous on $\overline{\mathbb{D}}$ and $\mathcal{C}=g(\mathbb{T})$ is asymptotically conformal.

(2) $\log g^{\prime} \in \mathrm{VMOA}$ if and only if $g^{\prime} \in H^{1}$ and $\mathcal{C}$ is asymptotically smooth.

The proof of Theorem 3, which is carried over in Section 3, is based on a localizing technique which allows to consider a closed curve with self-intersections as a finite union of Jordan arcs.

The next example shows that Theorem 3 is in a sense a strong generalization of the classical results mentioned in the introduction.

Example 3. Consider $g(z)=e^{\lambda z}$, where $\lambda \in(\pi, 2 \pi)$. Then $g$ is locally univalent and $g(\mathbb{T})$ is rectifiable and closed, but $g(\mathbb{D})$ is not simply connected nor $g(\mathbb{T})$ is Jordan. Moreover, $\log g^{\prime}(z)=\log \lambda+\lambda z$, and thus $\log g^{\prime} \in \mathcal{D} \subset$ VMOA with $\left\|\log g^{\prime}\right\|_{\mathcal{D}}=\lambda$. Therefore Part (2) in Theorem 3 ensures that $g(\mathbb{T})$ is asymptotically smooth. 

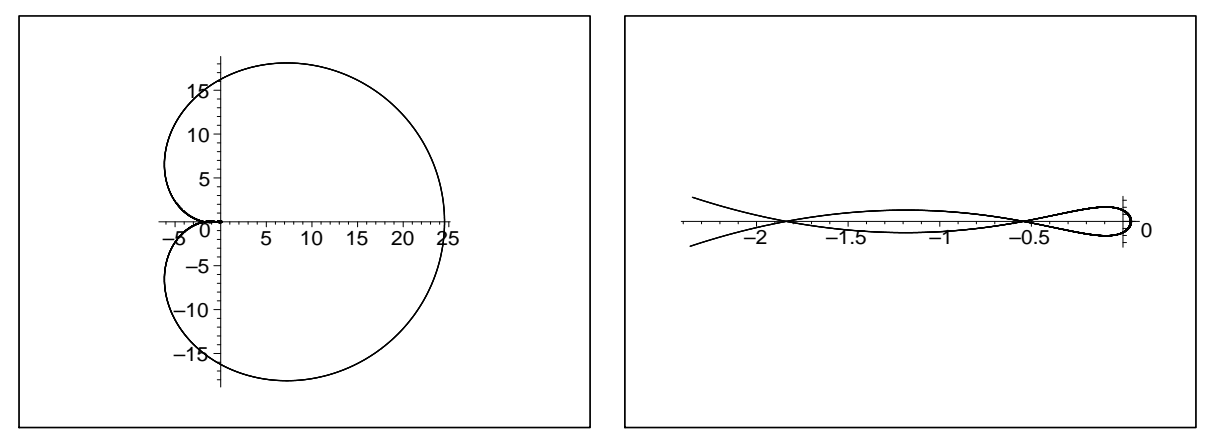

Figure 1. The left-hand picture corresponds to the curve $g(\mathbb{T})$ for $\lambda=3.2$. The right-hand picture shows the two loops near the origin which are too flat to be seen in the left-hand one.

In general, $\log g^{\prime} \in \mathcal{D}$ whenever, $\log g^{\prime}$ is a polynomial. For example, let $g$ be defined by

$$
\log g^{\prime}(z)=\lambda \sum_{k=1}^{200} \frac{1+i}{\sqrt{k}} z^{k}, \quad \lambda \in \mathbb{C} .
$$

Then $g$ is locally univalent, and Becker's univalence criterion [19, Theorem 1.11] shows that $g$ is univalent when $|\lambda|$ is small enough. As mentioned, $\log g^{\prime} \in \mathcal{D}$ and thus $g(\mathbb{T})$ is a rectifiable closed curve by Theorem 1 , so $g(\mathbb{T})$ is asymptotically smooth for all $\lambda \in \mathbb{C}$ by Part (2) in Theorem 3.

We next give an example of a bounded conformal map such that the boundary of the image domain is a non-rectifiable asymptotically conformal curve.

Example 4. Let $g$ be a conformal map of $\mathbb{D}$ onto the inner domain $\Omega$ of the Jordan curve given in Figure 2. The lower curve joining the points -1 and 1 to the origin is $\Im z=(\Re z)^{2} \cos \left(\pi /(\Re z)^{2}\right)$, and the upper curve is a part of a circle centered on $i / 2 \pi$. It is easy to see that $g(\mathbb{T})$ is not rectifiable and thus $\log g^{\prime} \notin$ VMOA by Part (2) in Theorem 1. However, a geometric reasoning shows that $\log g^{\prime} \in \mathcal{B}_{0}$ by [20, Theorem 1].

Next, we exhibit one more example of a locally univalent map $g$ such that $g(\mathbb{T})$ is asymptotically smooth.

Example 5. Let $\mu(z)=\log (3 /(1-z))$, and let $\lambda \in \mathbb{C}$ such that $\mu(z) \neq \lambda$ for all $z \in \mathbb{D}$. Consider $g(z):=(z-1)(\mu(z))^{\lambda}$. Then $g$ is locally univalent as $g^{\prime}(z)=(\mu(z))^{\lambda-1}(\mu(z)-\lambda)$, and also $\log g^{\prime} \in \mathcal{D}$ since

$$
\frac{g^{\prime \prime}(z)}{g^{\prime}(z)}=\frac{1}{1-z}\left(\frac{\lambda-1}{\log \frac{3}{1-z}}+\frac{1}{\log \frac{3}{1-z}-\lambda}\right)=\frac{\lambda}{1-z}\left(\frac{\log \frac{3}{1-z}-\lambda+1}{\log \frac{3}{1-z}\left(\log \frac{3}{1-z}-\lambda\right)}\right) .
$$




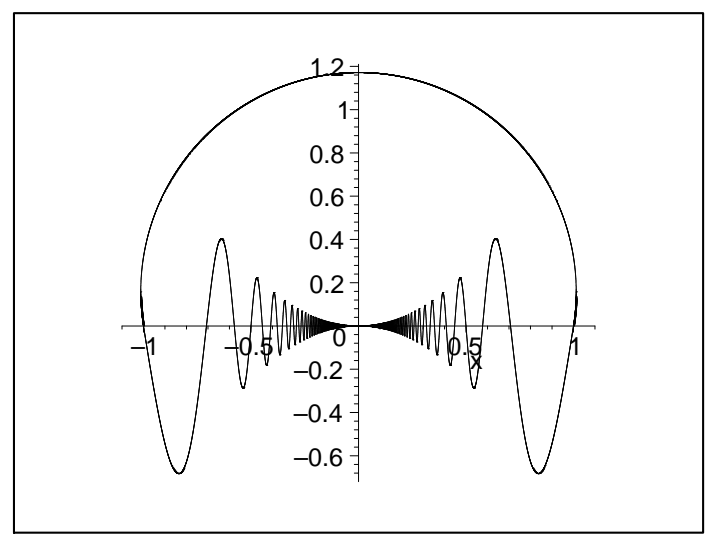

Figure 2. The domain $\Omega=g(\mathbb{D})$ of Example 4 .

Moreover, Becker's univalence criterion [19, Theorem 1.11] shows that $g$ is univalent if $|\lambda|$ is sufficiently small. We deduce that $g(\mathbb{T})$ is asymptotically smooth by Part (2) in Theorem 3.

The rest of our results concern the question of when $\log g^{\prime}$ belongs to the Dirichlet space $\mathcal{D}$. In this case, in contrary to VMOA and $\mathcal{B}_{0}$, there are no known geometric characterizations for $g(\mathbb{T})$ even in the case when $g$ is conformal. Next theorem is an attempt to establish such a characterization. Indeed, Jones [14] suggested the statement involving (1.8) to the authors and conjectured that the converse implication also holds.

Theorem 4. Let $g \in \mathcal{H}(\mathbb{D})$ be locally univalent. If $\log g^{\prime} \in \mathcal{D}$, then $\mathcal{C}=g(\mathbb{T})$ is asymptotically smooth and satisfies

$$
\int_{\mathbb{T}} \int_{\mathbb{T}} \frac{l\left(g\left(z_{1}\right), g\left(z_{2}\right)\right)-\left|g\left(z_{1}\right)-g\left(z_{2}\right)\right|}{\left|g\left(z_{1}\right)-g\left(z_{2}\right)\right|^{3}}\left|g^{\prime}\left(z_{1}\right)\right|\left|g^{\prime}\left(z_{2}\right)\right|\left|d z_{1}\right|\left|d z_{2}\right|<\infty .
$$

In particular, if $g$ is conformal and $\log g^{\prime} \in \mathcal{D}$, then

$$
\int_{\mathcal{C}} \int_{\mathcal{C}} \frac{l\left(w_{1}, w_{2}\right)-\left|w_{1}-w_{2}\right|}{\left|w_{1}-w_{2}\right|^{3}}\left|d w_{1}\right|\left|d w_{2}\right|<\infty .
$$

If $g$ is conformal and $\log g^{\prime} \in \mathcal{D}$, then $\mathcal{C}=g(\mathbb{T})$ is asymptotically smooth by [20, Theorem 2], and therefore

$$
\varepsilon\left(w_{1}, w_{2}\right):=\frac{l\left(w_{1}, w_{2}\right)}{\left|w_{1}-w_{2}\right|}-1 \rightarrow 0, \quad \text { as } \quad\left|w_{1}-w_{2}\right| \rightarrow 0 .
$$

Hence the integral condition in equation (1.8) says that the errors $\varepsilon\left(w_{1}, w_{2}\right)$ somehow add up to a finite quantity.

The locally univalent functions $g$ defined in Examples 2, 3 and 5 satisfy $\log g^{\prime} \in \mathcal{D}$, and therefore Theorem 4 implies that the double integral condition (1.7) must be satisfied for each of these functions. 
The next theorem contains two different characterizations of when a locally univalent function $g$ satisfies $\log g^{\prime} \in \mathcal{D}$.

Theorem 5. Let $g \in \mathcal{H}(\mathbb{D})$ be locally univalent such that $\mathcal{C}=g(\mathbb{T})$ is rectifiable and let $\omega(s)=g(\psi(s)), 0 \leq s \leq l$, be the arc length parametrization of $\mathcal{C}$. Then the following assertions are equivalent:

(1) $\log g^{\prime} \in \mathcal{D}$;

(2) $\log g^{\prime} \in H^{2}$ and

$$
\int_{\mathbb{T}} \int_{\mathbb{T}} \frac{\left(\arg g^{\prime}(z)-\arg g^{\prime}(\zeta)\right)^{2}}{|z-\zeta|^{2}}|d z||d \zeta|<\infty ;
$$

(3) $\log g^{\prime} \in H^{2}$ and

$$
\int_{\mathbb{T}} \int_{\mathbb{T}} \frac{\left(\arg \omega^{\prime}(s)-\arg \omega^{\prime}(\sigma)\right)^{2}}{|z-\zeta|^{2}}|d z||d \zeta|<\infty,
$$

where $z=\psi(s)$ and $\zeta=\psi(\sigma)$.

If $\log g^{\prime} \in \mathcal{D}$, then Part (3) in Theorem 2 implies that the tangent at $g(z) \in \mathcal{C}$, with the angle $\arg \left(z g^{\prime}(z)\right)$, exists for all $z \in \mathbb{T}$ outside of a possible exceptional set of zero logarithmic capacity. Moreover, the term $\left(\arg \omega^{\prime}(s)-\arg \omega^{\prime}(\sigma)\right)^{2}$ in $(1.10)$ is a strictly geometric quantity because it refers only to the geometrically defined arc length and tangent angle. However, $z=\psi(s)$ and $\zeta=\psi(\sigma)$ refer to the conformal map which is not a geometrically defined quantity, and therefore none of the characterizations in Theorem 5 is purely geometric.

In the next corollary the integral in (1.12) contains only geometric quantities, but this is no longer the case of the additional assumption (1.11).

Corollary 6. Let $g \in \mathcal{H}(\mathbb{D})$ be locally univalent such that $\mathcal{C}=g(\mathbb{T})$ is rectifiable, and let $\omega(s)=g(\psi(s)), 0 \leq s \leq l$, be the arc length parametrization of $\mathcal{C}$. If $\log g^{\prime} \in \mathrm{VMOA}$ and there exists $c>0$ such that

$$
c \leq\left|g^{\prime}(z)\right| \leq \frac{1}{c}
$$

for almost all $z \in \mathbb{T}$, then $\log g^{\prime} \in \mathcal{D}$ if and only if

$$
\int_{0}^{l} \int_{0}^{l}\left(\frac{\arg \omega^{\prime}(s)-\arg \omega^{\prime}(\sigma)}{s-\sigma}\right)^{2} d s d \sigma<\infty .
$$

We give one more example related to Dirichlet functions.

Example 6. The function $\log g_{z_{k}}^{\prime}(z):=\log \left(1 /\left(1-\bar{z}_{k} z\right)\right)$ satisfies

$$
\left\|\log g_{z_{k}}^{\prime}\right\|_{\mathcal{D}}^{2}=\frac{1}{\pi} \int_{\mathbb{D}} \frac{\left|z_{k}\right|}{\left|1-\bar{z}_{k} z\right|^{2}} d A(z)=\log \frac{1}{1-\left|z_{k}\right|^{2}}
$$


for all $z_{k} \in \mathbb{D}$. Since $\mathcal{D}$ is linear, it follows that

$$
\log g^{\prime}(z):=\log \left(\prod_{k} \frac{1}{\left(1-\bar{z}_{k} z\right)^{\lambda_{k}}}\right)=\sum_{k} \lambda_{k} \log \frac{1}{1-\bar{z}_{k} z}=\sum_{k} \lambda_{k} \log g_{z_{k}}^{\prime}
$$

belongs to $\mathcal{D}$, if

$$
\sum_{k}\left|\lambda_{k}\right| \sqrt{\log \frac{1}{1-\left|z_{k}\right|}}<\infty
$$

In this case the locally univalent function $g$ must satisfy (1.7), (1.9) and (1.10) by Theorems 4 and 5 .

Finally, we conclude our study concerning the Dirichlet space by considering Jordan curves whose smoothness is measured by a Hölder condition. More precisely, a Jordan curve $\mathcal{C}$ is said to belong to the class $\Lambda^{1, \alpha}, 0<\alpha<1$, if it has a continuously differentiable parametrization $\omega(t), 0 \leq t \leq 2 \pi$, such that $\omega^{\prime}$ satisfies the Hölder condition

$$
\left|\omega^{\prime}(t)-\omega^{\prime}(\tau)\right| \leq C_{1}|t-\tau|^{\alpha}, \quad t, \tau \in(0,2 \pi),
$$

for some positive constant $C_{1}$, and $\omega^{\prime}(t) \neq 0$ for all $t \in[0,2 \pi]$. By the KelloggWarschawski theorem [19, Theorem 3.6] we may use the conformal parametrization $\omega(t)=g\left(e^{i t}\right)$, and therefore (1.13) is equivalent to

$$
\left|\arg g^{\prime}\left(e^{i t}\right)-\arg g^{\prime}\left(e^{i \tau}\right)\right| \leq C_{2}\left|e^{i t}-e^{i \tau}\right|^{\alpha}, \quad t, \tau \in(0,2 \pi) .
$$

With this notation the following theorem holds.

Theorem 7. Let $g$ be a conformal map of $\mathbb{D}$ onto the inner domain of a Jordan curve $\mathcal{C}$.

(1) If $\mathcal{C} \in \Lambda^{1, \alpha}$ for $\alpha \in\left(\frac{1}{2}, 1\right)$, then $\log g^{\prime} \in \mathcal{D}$.

(2) There exists $g \in \mathcal{H}(\mathbb{D})$ conformal such that $g(\mathbb{T})=\mathcal{C} \in \Lambda^{1, \frac{1}{2}}$ but $\log g^{\prime} \notin \mathcal{D}$.

The remaining part of this paper is devoted to proofs of the results presented in this section.

\section{Proof of Theorem 1}

2.1. Proof of (1). Let $0<p<\infty$ and $-1<\alpha<\infty$ be arbitrary but fixed, and denote $f:=\log g^{\prime}$. We may assume that $f$ is continuous on $\overline{\mathbb{D}}$; if this is not the case, consider the dilatations $f_{t}(z)=f(t z), 0<t<1$, and let $t \rightarrow 1^{-}$at the end of the proof.

Since $f \in \mathcal{B}_{0}$, for a given $\varepsilon>0$ there exists $r \in(0,1)$ such that $\left|f^{\prime}(z)\right|\left(1-|z|^{2}\right) \leq$ $\varepsilon$ whenever $|z| \in[r, 1)$. Denote $D(0, r):=\{z:|z|<r\}$. It is well known that 
there exists a constant $C>0$, depending only on $p$ and $\alpha$, such that

$$
\begin{aligned}
I_{1}\left(e^{f}\right) & :=\int_{\mathbb{D} \backslash D(0, r)}\left|e^{f(z)}\right|^{p}\left(1-|z|^{2}\right)^{\alpha} d A(z) \\
& \leq C\left(\int_{\mathbb{D}}\left|e^{f(z)}\right|^{p}\left|f^{\prime}(z)\right|^{p}\left(1-|z|^{2}\right)^{p+\alpha} d A(z)+\left|e^{f(0)}\right|^{p}\right) \\
& \leq C \varepsilon^{p} I_{1}\left(e^{f}\right)+C\left(\int_{D(0, r)}\left|e^{f(z)}\right|^{p}\left|f^{\prime}(z)\right|^{p}\left(1-|z|^{2}\right)^{p+\alpha} d A(z)+\left|e^{f(0)}\right|^{p}\right) .
\end{aligned}
$$

Choosing $\varepsilon$ such that $C \varepsilon^{p}=1 / 2$, and fixing $r$ accordingly, we obtain $I_{1}\left(e^{f}\right)<\infty$ from which the assertion follows since $e^{f}=g^{\prime}$.

2.2. Proof of (2). As mentioned in the introduction, we give an alternative proof for this known implication $[21,(3.5)]$ for convenience of the reader. Let $0<p<\infty$ be arbitrary but fixed, and assume $f=\log g^{\prime} \in$ VMOA. The Hardy-Stein-Spencer identity states that

$$
\|h\|_{H^{p}}^{p}=\frac{p^{2}}{2 \pi} \int_{\mathbb{D}}|h(z)|^{p-2}\left|h^{\prime}(z)\right|^{2} \log \frac{1}{|z|} d A(z)+|h(0)|^{p}
$$

for all $0<p<\infty$ and $h \in \mathcal{H}(\mathbb{D})$, see for example [22, p. 126]. Therefore it suffices to show that there exists $r \in(0,1)$ such that

$$
I_{2}\left(e^{f}\right):=\int_{\mathbb{D} \backslash D(0, r)}\left|e^{f(z)}\right|^{p}\left|f^{\prime}(z)\right|^{2} \log \frac{1}{|z|} d A(z)<\infty .
$$

As in Part (1), we may assume that $f$ is continuous on $\overline{\mathbb{D}}$. By Carleson's theorem [6] (see also [11, p. 157]) there exists $C_{1}>0$, depending only on $p$, such that

$$
\begin{aligned}
I_{2}\left(e^{f}\right) & \leq C_{1} \sup _{I} \frac{1}{|I|} \int_{S(I) \backslash D(0, r)}\left|f^{\prime}(z)\right|^{2} \log \frac{1}{|z|} d A(z)\left\|e^{f}\right\|_{H^{p}}^{p} \\
& \leq 2 C_{1} \sup _{|I|<1-r} \frac{1}{|I|} \int_{S(I)}\left|f^{\prime}(z)\right|^{2} \log \frac{1}{|z|} d A(z)\left\|e^{f}\right\|_{H^{p}}^{p} .
\end{aligned}
$$

It is well known that

$$
C_{2}^{-1}\|h\|_{\mathrm{BMOA}}^{2} \leq \sup _{I} \frac{1}{|I|} \int_{S(I)}\left|h^{\prime}(z)\right|^{2} \log \frac{1}{|z|} d A(z) \leq C_{2}\|h\|_{\mathrm{BMOA}}^{2}, \quad h \in \mathcal{H}(\mathbb{D}),
$$

for some constant $C_{2}>0$, see for instance [12, Lemma 3.3, p. 231]). This combined with the Hardy-Spencer-Stein identity (2.1) and (2.2) yields

$$
\begin{aligned}
I_{2}\left(e^{f}\right) \leq & C_{1} p^{2} \sup _{|I|<1-r} \frac{1}{|I|} \int_{S(I)}\left|f^{\prime}(z)\right|^{2} \log \frac{1}{|z|} d A(z) I_{2}\left(e^{f}\right) \\
& +2 C_{1} C_{2}\|f\|_{\mathrm{BMOA}}^{2} I_{3}\left(e^{f}\right),
\end{aligned}
$$


where

$$
I_{3}\left(e^{f}\right)=\frac{p^{2}}{2} \int_{D(0, r)}\left|e^{f(z)}\right|^{p}\left|f^{\prime}(z)\right|^{2} \log \frac{1}{|z|} d A(z)+\left|e^{f(0)}\right|^{p} .
$$

Since $f \in \mathrm{VMOA}$, we may fix $r \in(0,1)$ sufficiently large such that

$$
\sup _{|I|<1-r} \frac{1}{|I|} \int_{S(I)}\left|f^{\prime}(z)\right|^{2} \log \frac{1}{|z|} d A(z) \leq \frac{1}{2 C_{1} p^{2}},
$$

and it follows that

$$
I_{2}\left(e^{f}\right) \leq 4 C_{1} C_{2}\|f\|_{\mathrm{BMOA}}^{2} I_{3}\left(e^{f}\right)<\infty .
$$

2.3. Proof of (3). We will show that for $f \in \mathcal{D}$ the function $h:=e^{f}$ satisfies

$$
\int_{\mathbb{D}}|h(z)|^{p-2}\left|h^{\prime}(z)\right|^{2}\left(\log \frac{1}{|z|}\right)^{\alpha} d A(z)<\infty
$$

for all $0<p<\infty$ and $0<\alpha<\infty$. Part (3) in Theorem 1 then follows by choosing $f=\log g^{\prime}$.

To prove (2.3), note first that, as $-\log r \leq(1-r) / r$ for all $r \in(0,1)$, it suffices to show

$$
I_{4}(h):=\int_{\mathbb{D} \backslash D\left(0, r_{0}\right)}|h(z)|^{p-2}\left|h^{\prime}(z)\right|^{2}(1-|z|)^{\alpha} d A(z)<\infty
$$

for $r_{0}:=1-e^{-\frac{p}{\alpha}} \in(0,1)$. Write $f(z)=\sum_{n=0}^{\infty} a_{n} z^{n}, z=r e^{i t}$. Since $f \in \mathcal{D}$, equality (1.2) implies that there exists $N_{\alpha, p} \in \mathbb{N}$ such that $\sum_{n=N_{\alpha, p}}^{\infty} n\left|a_{n}\right|^{2}<\alpha / p$. By the Cauchy-Schwarz inequality this yields

$$
\left(\sum_{n=N_{\alpha, p}}^{\infty}\left|a_{n}\right| r^{n}\right)^{2} \leq\left(\sum_{n=N_{\alpha, p}}^{\infty} n\left|a_{n}\right|^{2}\right)\left(\sum_{n=N_{\alpha, p}}^{\infty} \frac{r^{2 n}}{n}\right) \leq \frac{\alpha}{p} \log \frac{1}{1-r}, \quad 0<r<1,
$$

and hence

$$
\sum_{n=N_{\alpha, p}}^{\infty}\left|a_{n}\right| r^{n} \leq\left(\log \frac{1}{(1-r)^{\frac{\alpha}{p}}}\right)^{\frac{1}{2}} \leq \log \frac{1}{(1-r)^{\frac{\alpha}{p}}}, \quad r \geq r_{0}
$$

But now

$$
|f(z)| \leq \sum_{n=0}^{N_{\alpha, p}-1}\left|a_{n}\right|+\sum_{n=N_{\alpha, p}}^{\infty}\left|a_{n}\right| r^{n}=: C+\sum_{n=N_{\alpha, p}}^{\infty}\left|a_{n}\right| r^{n}
$$

and therefore

$$
M_{\infty}\left(r, e^{f}\right)=\max _{|z|=r}\left|e^{f(z)}\right| \leq \frac{e^{C}}{(1-r)^{\frac{\alpha}{p}}}, \quad r \geq r_{0}
$$


It follows that

$$
\begin{aligned}
I_{4}(h) & \leq \int_{\mathbb{D} \backslash D\left(0, r_{0}\right)} M_{\infty}^{p}\left(|z|, e^{f}\right)\left|f^{\prime}(z)\right|^{2}(1-|z|)^{\alpha} d A(z) \\
& \leq e^{p C} \int_{\mathbb{D} \backslash D\left(0, r_{0}\right)}\left|f^{\prime}(z)\right|^{2}(1-|z|)^{\alpha-\alpha} d A(z) \leq e^{p C} \pi\|f\|_{\mathcal{D}}^{2}<\infty .
\end{aligned}
$$

Remark. An application of the first inequality in (2.4) shows that the term $(\log 1 /|z|)^{\alpha}$ in $(1.4)$ can be replaced by $\exp \left(-\alpha(\log (1 /|z|))^{\frac{1}{2}}\right)$.

\section{Proof of Theorem 3}

Let $g$ be locally univalent such that $g(\mathbb{T})$ is a closed curve. We will prove the following assertions which are equivalent to the statements of Theorem 3 :

(1) $\log g^{\prime} \in \mathcal{B}_{0}$ if and only if $g$ is continuous on $\overline{\mathbb{D}}$ and there exists $\delta_{0}>0$ such that $g(I)$ is an asymptotically conformal Jordan arc for any interval $I$ on $\mathbb{T}$ with $|I|<\delta_{0}$.

(2) $\log g^{\prime} \in \mathrm{VMOA}$ if and only if $g^{\prime} \in H^{1}$ and there exists $\delta_{0}>0$ such that $g(I)$ is an asymptotically smooth Jordan arc for any interval $I$ on $\mathbb{T}$ with $|I|<\delta_{0}$.

3.1. Sufficiency. Let $\delta, \rho \in(0,1)$ such that $2 \delta+\rho<1$. Consider the circles $\mathbb{T}$, $\partial D(1+\rho, 1)$ and $\partial D\left(c_{ \pm}, r\right)$, where $c_{ \pm}=\frac{1+\rho}{2}(1 \pm i \tan \delta)$ and $r=\left|e^{i \delta}-c_{+}\right|$. The discs $D\left(c_{ \pm}, r\right)$ are contained in both $\mathbb{D}$ and $D(1+\rho, 1)$. Moreover, the circles $\partial D\left(c_{ \pm}, r\right)$ intersect $\mathbb{T}$ on the points $e^{ \pm i \delta}$, and the common points of $\partial D\left(c_{ \pm}, r\right)$ and $\partial D(1+\rho, 1)$ are the reflections of $e^{ \pm i \delta}$ with respect to the line $\Re z=\frac{1+\rho}{2}$. Let us call them $\gamma_{ \pm}$according to the sign of their imaginary parts. Let $\Omega_{\delta, \rho}$ be the Jordan domain formed by the shortest four circular arcs connecting $e^{ \pm i \delta}$ and $\gamma_{ \pm}$on these four circles. Let $\phi_{\delta, \rho}$ be the conformal map of $\mathbb{D}$ onto $\Omega_{\delta, \rho}$ such that $\phi_{\delta, \rho}(0)=\frac{1+\rho}{2}$ and $\phi_{\delta, \rho}^{\prime}(0)>0$.
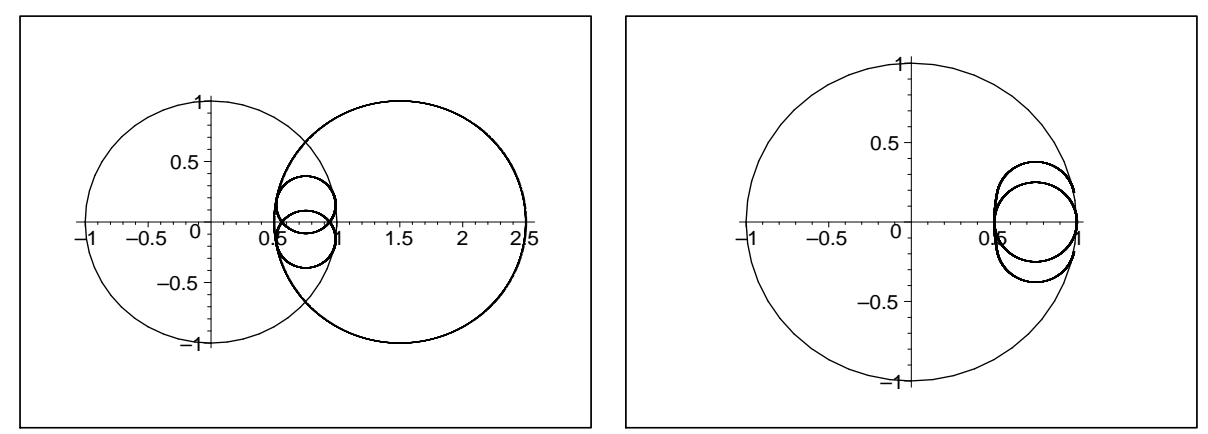

Figure 3. The four circles and $\phi_{3 / 16,1 / 2}(\mathbb{T})$ with $\partial D(3 / 4,1 / 4)$. 
Lemma 8. Let $0<p<\infty$ and let $\delta, \rho \in(0,1)$ such that $2 \delta+\rho<1$. Then $\phi_{\delta, \rho}$ satisfies $\left(\log \phi_{\delta, \rho}^{\prime}\right)^{\prime} \in H^{p}, \phi_{\delta, \rho}^{\prime \prime} \in H^{p}$, and

$$
\int_{\mathbb{D}}\left|\frac{\phi_{\delta, \rho}^{\prime \prime}(z)}{\phi_{\delta, \rho}^{\prime}(z)}\right|^{p} d A(z) \rightarrow 0, \quad \delta \rightarrow 0^{+} .
$$

Proof. It suffices to consider the values $1<p<\infty$. Note first that $\phi_{\delta, \rho} \rightarrow$ $\frac{1+\rho}{2}+\frac{1-\rho}{2} z$, as $\delta \rightarrow 0^{+}$, and therefore $\phi_{\delta, \rho}^{\prime \prime} / \phi_{\delta, \rho}^{\prime} \rightarrow 0$, as $\delta \rightarrow 0^{+}$, locally uniformly in $\mathbb{D}$ by $\left[22\right.$, Theorem 1.8]. Applying [26] to $\phi_{\delta, \rho}^{*}:=\phi_{\delta, \rho}-\frac{1+\rho}{2}$, we obtain

$$
\int_{\mathbb{T}}\left|\phi_{\delta, \rho}^{\prime}(z)-\frac{1-\rho}{2}\right|^{p}|d z| \rightarrow 0, \quad \delta \rightarrow 0^{+} .
$$

Therefore there exists $C_{1}>0$, depending only on $\rho$ and $p$, such that

$$
\int_{\mathbb{T}}\left|\phi_{\delta, \rho}^{\prime}(z)\right|^{p}|d z| \leq C_{1}
$$

for all $\delta \in(0,(1-\rho) / 2)$, and thus $\phi_{\delta, \rho}^{\prime} \in H^{p}$. By [19, p. 43], the curvature of $\phi_{\delta, \rho}(\mathbb{T})$ at $\phi_{\delta, \rho}(z)$ satisfies

$$
\kappa\left(\phi_{\delta, \rho}(z)\right)=\frac{1}{\left|\phi_{\delta, \rho}^{\prime}(z)\right|}\left(1+\Re\left(z \frac{\phi_{\delta, \rho}^{\prime \prime}(z)}{\phi_{\delta, \rho}^{\prime}(z)}\right)\right)
$$

when $z$ is none of the preimages of the four exceptional boundary points where either of the circles $\mathbb{T}$ and $\partial D(1+\rho, 1)$ intersects $\partial D\left(c_{ \pm}, r\right)$. By the construction, $0 \leq \kappa\left(\phi_{\delta, \rho}(z)\right) \leq 3 /(1-\rho)=: C_{2}$ for all $\delta \in(0,(1-\rho) / 2)$. It follows that

$$
\begin{aligned}
\int_{\mathbb{T}}\left|\Re\left(z \frac{\phi_{\delta, \rho}^{\prime \prime}(z)}{\phi_{\delta, \rho}^{\prime}(z)}\right)\right|^{p}|d z| & \leq 2^{p-1}+2^{p-1} \int_{\mathbb{T}}\left|1+\Re\left(z \frac{\phi_{\delta, \rho}^{\prime \prime}(z)}{\phi_{\delta, \rho}^{\prime}(z)}\right)\right|^{p}|d z| \\
& \leq 2^{p-1}+2^{p-1} C_{2}^{p} \int_{\mathbb{T}}\left|\phi_{\delta, \rho}^{\prime}(z)\right|^{p}|d z|,
\end{aligned}
$$

and hence [12, p. 104; Theorem 1.5] and (3.2) yield

$$
\int_{\mathbb{T}}\left|\frac{\phi_{\delta, \rho}^{\prime \prime}(z)}{\phi_{\delta, \rho}^{\prime}(z)}\right|^{p}|d z| \leq 2^{p}+2^{p} C_{2}^{p} C_{1},
$$

that is, $\left(\log \phi_{\delta, \rho}^{\prime}\right)^{\prime} \in H^{p}$. As both $\phi_{\delta, \rho}^{\prime}$ and $\left(\log \phi_{\delta, \rho}^{\prime}\right)^{\prime}$ belong to $H^{p}$ for all $0<p<$ $\infty$, the Cauchy-Schwarz inequality yields $\phi_{\delta, \rho}^{\prime \prime} \in H^{p}$ for all $0<p<\infty$ as claimed.

To see (3.1), let $\varepsilon>0$ be given and choose $r_{\varepsilon} \in(0,1)$ such that

$$
\int_{\mathbb{D} \backslash D\left(0, r_{\varepsilon}\right)}\left|\frac{\phi_{\delta, \rho}^{\prime \prime}(z)}{\phi_{\delta, \rho}^{\prime}(z)}\right|^{p} d A(z)<\frac{\varepsilon}{2} .
$$

By the uniform convergence there exists $\delta_{0} \in(0,(1-\rho) / 2)$ such that

$$
\int_{D\left(0, r_{\varepsilon}\right)}\left|\frac{\phi_{\delta, \rho}^{\prime \prime}(z)}{\phi_{\delta, \rho}^{\prime}(z)}\right|^{p} d A(z)<\frac{\varepsilon}{2}
$$

for all $\delta \in\left(0, \delta_{0}\right)$. These inequalities yield (3.1). 
Remarks. (1) The conformal map $\phi_{\delta, \rho}$ satisfies

$$
\phi_{\delta, \rho}^{\prime} \in H^{p} \Rightarrow\left(\log \phi_{\delta, \rho}^{\prime}\right)^{\prime} \in H^{p}, \quad 1<p<\infty,
$$

by the proof of Lemma 8. This implication is not true in general, i.e., $e^{f} \in H^{p} \not \Rightarrow$ $f^{\prime} \in H^{p}$, as the function $f_{\lambda}(z):=-\lambda \log (1-z)$ with $\lambda \in(0,1 / p)$ shows. Note that, setting $\phi_{\lambda}^{\prime}:=e^{f_{\lambda}}$, the curvature of $\phi_{\lambda}(\mathbb{T})$ at $\phi_{\lambda}(z)$ equals to $|1-z|^{\lambda}\left(1-\frac{\lambda}{2}\right)$ when $z \in \mathbb{T} \backslash\{1\}$.

(2) By Lemma $8, \phi_{\delta, \rho}^{\prime \prime} \in H^{p}$ for all $0<p<\infty$. It follows that there exists a constant $C>0$, depending only on $p$, such that $M_{\infty}\left(r, \phi_{\delta, \rho}^{\prime \prime}\right) \leq C(1-r)^{-\frac{1}{p}}$. Choosing $p>1$, this implies that $\phi_{\delta, \rho}^{\prime}$ is continuous on $\overline{\mathbb{D}}$.

Lemma 9. Let $g \in \mathcal{H}(\mathbb{D})$ be a locally univalent function, and denote $\tilde{g}_{\delta, \rho}:=$ $g \circ \phi_{\delta, \rho}$. Assume $X \in\{\mathrm{VMOA}, \mathcal{D}\}$. If $\log g^{\prime} \in \mathcal{B}_{0} \cap X$, then for any $C>0$ there exist $\rho \in(0,1)$ and $\delta_{0} \in(0,(1-\rho) / 2)$ such that

$$
\left\|\log \tilde{g}_{\delta, \rho}^{\prime}\right\|_{\mathcal{B}}<C \quad \text { and } \quad \log \tilde{g}_{\delta, \rho}^{\prime} \in \mathcal{B}_{0} \cap X
$$

for all $\delta \in\left(0, \delta_{0}\right)$.

Proof. To establish the first property in (3.5), assume $\log g^{\prime} \in \mathcal{B}_{0}$, and fix $\rho \in$ $(0,1)$ such that $\left|\left(\log g^{\prime}\right)^{\prime}(z)\right|\left(1-|z|^{2}\right)<C / 2$ for all $z \in \mathbb{D}$ with $|z| \in(\rho, 1)$. Then

$$
\left(\log \tilde{g}_{\delta, \rho}^{\prime}\right)^{\prime}(z)=\frac{\tilde{g}_{\delta, \rho}^{\prime \prime}(z)}{\tilde{g}_{\delta, \rho}^{\prime}(z)}=\frac{g^{\prime \prime}\left(\phi_{\delta, \rho}(z)\right)}{g^{\prime}\left(\phi_{\delta, \rho}(z)\right)} \phi_{\delta, \rho}^{\prime}(z)+\frac{\phi_{\delta, \rho}^{\prime \prime}(z)}{\phi_{\delta, \rho}^{\prime}(z)},
$$

and hence the Schwarz-Pick lemma yields

$$
\begin{aligned}
\left\|\log \tilde{g}_{\delta, \rho}^{\prime}\right\|_{\mathcal{B}} & \leq \sup _{z \in \mathbb{D}}\left|\left(\log g^{\prime}\right)^{\prime}\left(\phi_{\delta, \rho}(z)\right)\right|\left(1-\left|\phi_{\delta, \rho}(z)\right|^{2}\right)+\left\|\log \phi_{\delta, \rho}^{\prime}\right\|_{\mathcal{B}} \\
& \leq \frac{C}{2}+C_{1}\left\|\log \phi_{\delta, \rho}^{\prime}\right\|_{\mathcal{D}}
\end{aligned}
$$

for some constant $C_{1}>0$, independent of $\delta$ and $\rho$. By Lemma 8, there exists $\delta_{0} \in(0,(1-\rho) / 2)$ such that $\left\|\log \phi_{\delta, \rho}^{\prime}\right\|_{\mathcal{D}}<C /\left(2 C_{1}\right)$ for all $\delta \in\left(0, \delta_{0}\right)$. It follows that $\left\|\log \tilde{g}_{\delta, \rho}^{\prime}\right\|_{\mathcal{B}}<C$ for all $\delta \in\left(0, \delta_{0}\right)$.

To prove the second property in (3.5), recall that $\log \phi_{\delta, \rho}^{\prime} \in \mathcal{D}$ by Lemma 8. Since $\mathcal{D} \subset \mathrm{VMOA} \subset \mathcal{B}_{0}$, the identity (3.6) implies that it suffices to show $f \circ \phi_{\delta, \rho} \in X$ for any $f \in X \in\left\{\mathcal{B}_{0}, \mathrm{VMOA}, \mathcal{D}\right\}$. These three cases are considered separately.

Let $f \in \mathcal{B}_{0}$, and consider

$$
I(z):=\left|\left(f \circ \phi_{\delta, \rho}\right)^{\prime}(z)\right|\left(1-|z|^{2}\right)=\left|f^{\prime}\left(\phi_{\delta, \rho}(z)\right)\right|\left(1-\left|\phi_{\delta, \rho}(z)\right|^{2}\right) \frac{\left|\phi_{\delta, \rho}^{\prime}(z)\right|\left(1-|z|^{2}\right)}{1-\left|\phi_{\delta, \rho}(z)\right|^{2}} .
$$

Since $\phi_{\delta, \rho}^{\prime \prime} \in H^{p}$ for all $0<p<\infty$ by Lemma $8, \phi_{\delta, \rho}^{\prime} \in H^{\infty}$, and hence, in particular, $\phi_{\delta, \rho} \in \mathcal{B}_{0}$. This and the assumption $f \in \mathcal{B}_{0}$ yields $I(z) \rightarrow 0$, as $|z| \rightarrow 1^{-}$. 
Let now $f \in \mathrm{VMOA}$, and consider

$$
T_{k}(f):=\frac{1}{\left|I_{k}\right|} \int_{S\left(I_{k}\right)}\left|\left(f \circ \phi_{\delta, \rho}\right)^{\prime}(z)\right|^{2}\left(1-|z|^{2}\right) d A(z),
$$

where $\left\{I_{k}\right\}$ is a sequence of $\operatorname{arcs}$ on $\mathbb{T}$ such that $\left|I_{k}\right| \rightarrow 0$, as $k \rightarrow \infty$, and $S\left(I_{k}\right):=\left\{z: 1-\left|I_{k}\right| \leq|z|, z /|z| \in I_{k}\right\}$. Let $\xi_{k} \in \mathbb{T}$ be the midpoint of $I_{k}$, and set $a_{k}:=\left(1-\left|I_{k}\right|\right) \xi_{k}$. Without loss of generality, assume $\phi_{\delta, \rho}\left(a_{k}\right) \rightarrow \zeta \in \overline{\mathbb{D}}$, as $k \rightarrow \infty$. To prove $f \circ \phi_{\delta, \rho} \in \mathrm{VMOA}$, it suffices to show that $T_{k}(f) \rightarrow 0$, as $k \rightarrow \infty$. If $\left|\phi_{\delta, \rho}\left(a_{k}\right)\right| \nrightarrow 1$, as $k \rightarrow \infty$, then there exist $r_{0} \in(0,1)$ and $N_{r_{0}} \in \mathbb{N}$ such that $\left|\phi_{\delta, \rho}(z)\right| \leq r_{0} \in(0,1)$ for all $z \in S\left(I_{k}\right)$ when $k \geq N_{r_{0}}$. Moreover, $\phi_{\delta, \rho}^{\prime} \in H^{\infty}$ by Lemma 8, and therefore

$$
\begin{aligned}
T_{k}(f) & \leq \frac{M_{\infty}^{2}\left(r_{0}, f^{\prime}\right)}{\left|I_{k}\right|} \int_{S\left(I_{k}\right)}\left|\phi_{\delta, \rho}^{\prime}(z)\right|^{2}\left(1-|z|^{2}\right) d A(z) \\
& \leq M_{\infty}^{2}\left(r_{0}, f^{\prime}\right) C\left\|\phi_{\delta, \rho}^{\prime}\right\|_{H^{\infty}}^{2}\left|I_{k}\right|^{2} \rightarrow 0, \quad k \rightarrow \infty .
\end{aligned}
$$

Assume now that $\left|\phi_{\delta, \rho}\left(a_{k}\right)\right| \rightarrow 1$, as $k \rightarrow \infty$. Since $\left|I_{k}\right|^{-1} \leq 10\left|\varphi_{a_{k}}^{\prime}(z)\right|$ for all $z \in S\left(I_{k}\right)$, and $1-r^{2} \leq-2 \log r$ for all $r \in(0,1]$, we have

$$
T_{k}(f) \leq 20 \int_{\mathbb{D}}\left|\left(f \circ \phi_{\delta, \rho}\right)^{\prime}(z)\right|^{2} \log \frac{1}{\left|\varphi_{a_{k}}(z)\right|} d A(z) .
$$

The Littlewood-Paley identity [12, Lemma 3.1, pp.228] (the case $p=2$ of the first formula in Section 2.1) along with the Littlewood's Subordination Principle [11, Theorem 1.7] yields

$$
\begin{aligned}
T_{k}(f) & \leq 5 \int_{\mathbb{T}}\left|\left(f \circ \phi_{\delta, \rho} \circ \varphi_{a_{k}}\right)(z)-f\left(\phi_{\delta, \rho}\left(a_{k}\right)\right)\right|^{2}|d z| \\
& \leq 5 \int_{\mathbb{T}}\left|f \circ \varphi_{\phi_{\delta, \rho}\left(a_{k}\right)}(z)-f\left(\phi_{\delta, \rho}\left(a_{k}\right)\right)\right|^{2}|d z| .
\end{aligned}
$$

Since $f \in \mathrm{VMOA}$ and $\left|\phi_{\delta, \rho}\left(a_{k}\right)\right| \rightarrow 1$, as $k \rightarrow \infty$, it follows that $T_{k}(f) \rightarrow 0$ as $k \rightarrow \infty$. It is well known that this implies $f \circ \phi_{\delta, \rho} \in$ VMOA.

If $f \in \mathcal{D}$, then clearly $\left\|f \circ \phi_{\delta, \rho}\right\|_{\mathcal{D}} \leq\|f\|_{\mathcal{D}}$, and thus $f \circ \phi_{\delta, \rho} \in \mathcal{D}$.

We are now ready to prove the sufficiency part of Theorem 3. To this end, assume $\log g^{\prime} \in \mathcal{B}_{0} \cap X$, where $X \in\left\{\right.$ VMOA, $\left.\mathcal{B}_{0}\right\}$. Then $g$ is continuous on $\overline{\mathbb{D}}$ by Part (1) in Theorem 1, and further $g^{\prime} \in H^{1}$ if $X=$ VMOA by Part (2) in Theorem 1. Furthermore, by Lemma 9 there exist $\rho \in(0,1)$ and $\delta_{0} \in(0,(1-\rho) / 2)$ such that

$$
\left\|\log \tilde{g}_{\delta, \rho}^{\prime}\right\|_{\mathcal{B}}<1 \quad \text { and } \quad \log \tilde{g}_{\delta, \rho}^{\prime} \in \mathcal{B}_{0} \cap X
$$

for all $\delta \in\left(0, \delta_{0}\right)$. Becker's univalence criterion [19, Theorem 1.11] shows that $\tilde{g}_{\delta, \rho}$ is univalent, and since $g(\mathbb{T})$ is closed by the assumption, $\tilde{g}_{\delta, \rho}(\mathbb{T})$ is a Jordan curve. Hence $\tilde{g}_{\delta, \rho}(\mathbb{T})$ is asymptotically conformal by [20, Theorem 1]. Moreover, $\tilde{g}_{\delta, \rho}(\mathbb{T})$ is asymptotically smooth by [20, Theorem 2] if $X=$ VMOA. 
Let $I$ be an arc on $\mathbb{T}$ such that $|I|<2 \delta_{0}$, and let $\zeta=e^{i t} \in \mathbb{T}$ be the midpoint of $I$. Set $\phi_{I}(z):=e^{i t} \phi_{|I|, \rho}(z)$ and define $g_{I}:=g \circ \phi_{I}$. Since the spaces $\mathcal{B}, \mathcal{B}_{0}$ and VMOA are invariant under rotations, the Jordan curve $g_{I}(\mathbb{T})$ must have the same properties as $\tilde{g}_{|I|, \rho}(\mathbb{T})$. But now $g(I)=g\left(\phi_{I}(\mathbb{T}) \cap \mathbb{T}\right) \subset g_{I}(\mathbb{T})$ and therefore $g(I)$ has the desired properties according to $X$.

3.2. Necessity. (1) Assume that $g$ is a locally univalent function on $\mathbb{D}$, continuous on $\overline{\mathbb{D}}$, and that there exists $\delta_{0}>0$ such that $g(I)$ is an asymptotically conformal Jordan arc for any interval $I$ on $\mathbb{T}$ with $|I|<\delta_{0}$. Let $\tau_{\nu} \in \mathbb{R}$ such that the intervals $I_{\nu}:=\left\{e^{i t}: \tau_{\nu} \leq t \leq \tau_{\nu+2}\right\}$ satisfy $\mathbb{T}=I_{0} \cup I_{2} \cup \cdots \cup I_{n-1}=I_{1} \cup I_{3} \cup \cdots \cup I_{n}$ and $\left|I_{\nu}\right|=\left|I_{\nu+1}\right|=: \delta<\delta_{0}$ for all $\nu=0, \ldots, n-1$. We aim to show that $f=\log g^{\prime} \in \mathcal{B}_{0}$. To see this, let $\zeta \in \mathbb{T}$ be arbitrary but fixed and let $w_{k} \rightarrow \zeta$, as $k \rightarrow \infty$. Let $I_{\zeta}$ be the interval on $\mathbb{T}$ with midpoint $\zeta$ such that $\left|I_{\zeta}\right|=\delta / 2$. Then there exists $\nu \in\{0, \ldots, n\}$ such that $I_{\zeta} \subset I_{\nu}$. Set $\phi_{\nu}(z):=e^{i \tau_{\nu+1}} \phi_{\delta / 2, \rho}(z)$ so that $\mathbb{T} \cap \phi_{\nu}(\mathbb{T})=I_{\nu}$, where $\rho$ is chosen to be large enough such that $g_{\nu}:=g \circ \phi_{\nu}$ is univalent by Lemma 8 . Then $g_{\nu}(\mathbb{T})$ is an asymptotically conformal Jordan curve and hence $f_{\nu}:=\log g_{\nu}^{\prime} \in \mathcal{B}_{0}$ by [20, Theorem 1]. Moreover, there exists $N_{\nu} \in \mathbb{N}$ such that $w_{k} \in \phi_{\nu}(\mathbb{D})$ for all $k \geq N_{\nu}$. Let $z_{k} \in \mathbb{D}$ such that $\phi_{\nu}\left(z_{k}\right)=w_{k}$ for $k \geq N_{\nu}$. Then clearly $\left|z_{k}\right| \rightarrow 1^{-}$as $k \rightarrow \infty$, and since $\phi_{\nu}^{\prime} \in H^{\infty}$ by Lemma 8 , it follows that $1-\left|\phi_{\nu}\left(z_{k}\right)\right|^{2} \leq C\left(1-\left|z_{k}\right|\right)$ for all sufficiently large $k$ and for some constant $C>0$. This combined with (3.6) yields

$$
\begin{aligned}
\left|f^{\prime}\left(w_{k}\right)\right|\left(1-\left|w_{k}\right|^{2}\right) \leq & \left|f_{\nu}^{\prime}\left(z_{k}\right)\right| \frac{1-\left|\phi_{\nu}\left(z_{k}\right)\right|^{2}}{\left|\phi_{\nu}^{\prime}\left(z_{k}\right)\right|} \\
& +\left|\frac{\phi_{\nu}^{\prime \prime}\left(z_{k}\right)}{\phi_{\nu}^{\prime}\left(z_{k}\right)}\right|\left(1-\left|\phi_{\nu}\left(z_{k}\right)\right|^{2}\right) \\
\leq & \left|f_{\nu}^{\prime}\left(z_{k}\right)\right|\left(1-\left|z_{k}\right|^{2}\right) \frac{1-\left|\phi_{\nu}\left(z_{k}\right)\right|^{2}}{\left|\phi_{\nu}^{\prime}\left(z_{k}\right)\right|\left(1-\left|z_{k}\right|^{2}\right)} \\
& +C\left|\frac{\phi_{\nu}^{\prime \prime}\left(z_{k}\right)}{\phi_{\nu}^{\prime}\left(z_{k}\right)}\right|\left(1-\left|z_{k}\right|^{2}\right)
\end{aligned}
$$

for all sufficiently large $k$. As $f_{\nu} \in \mathcal{B}_{0}$ by the assumption and $\log \phi_{\nu}^{\prime} \in \mathcal{B}_{0}$ by Lemma 8, [19, Corollary 1.4] yields $\left|f^{\prime}\left(w_{k}\right)\right|\left(1-\left|w_{k}\right|^{2}\right) \rightarrow 0$ as $k \rightarrow \infty$. Since $\zeta \in \mathbb{T}$ was arbitrary, we deduce $\log g^{\prime} \in \mathcal{B}_{0}$.

(2) Assume that $g$ is a locally univalent function on $\mathbb{D}$ with $g^{\prime} \in H^{1}$ and that there exists $\delta_{0}>0$ such that $g(I)$ is an asymptotically smooth Jordan arc for any interval $I$ on $\mathbb{T}$ with $|I|<\delta_{0}$. With the same notation as in Part (1), we deduce that $g_{\nu}(\mathbb{T})$ is an asymptotically smooth Jordan curve, and thus $f_{\nu}=\log g_{\nu}^{\prime} \in$ VMOA for all $\nu=0, \ldots, n$ by [20, Theorem 2]. To prove $f=\log g^{\prime} \in \mathrm{VMOA}$, consider

$$
T_{k}(f):=\frac{1}{\left|J_{k}\right|} \int_{S\left(J_{k}\right)}\left|f^{\prime}(w)\right|^{2}\left(1-|w|^{2}\right) d A(w)
$$


where $\left\{J_{k}\right\}$ is a sequence of arcs on $\mathbb{T}$ such that $\left|J_{k}\right| \rightarrow 0$, as $k \rightarrow \infty$. Let $\xi_{k} \in \mathbb{T}$ be the midpoint of $J_{k}$. Without loss of generality, assume $\xi_{k} \rightarrow \zeta \in \mathbb{T}$, as $k \rightarrow \infty$. By the proof of Part (1), $I_{\zeta} \subset I_{\nu}$ for some $\nu \in\{0, \ldots, n\}$, and hence there exists $M_{\nu} \in \mathbb{N}$ such that $S\left(J_{k}\right) \subset \phi_{\nu}(\mathbb{D})$ for all $k \geq M_{\nu}$. Therefore

$$
\begin{aligned}
T_{k}(f) \leq & \frac{2}{\left|J_{k}\right|} \int_{\phi_{\nu}^{-1}\left(S\left(J_{k}\right)\right)}\left|f_{\nu}^{\prime}(z)\right|^{2}\left(1-\left|\phi_{\nu}(z)\right|^{2}\right) d A(z) \\
& +\frac{2}{\left|J_{k}\right|} \int_{\phi_{\nu}^{-1}\left(S\left(J_{k}\right)\right)}\left|\frac{\phi_{\nu}^{\prime \prime}(z)}{\phi_{\nu}^{\prime}(z)}\right|^{2}\left(1-\left|\phi_{\nu}(z)\right|^{2}\right) d A(z), \quad k \geq M_{\nu} .
\end{aligned}
$$

For each $k \geq M_{\nu}$ sufficiently large there exists an interval $L_{k}$ on $\mathbb{T}$ such that $\phi_{\nu}^{-1}\left(S\left(J_{k}\right)\right) \subset S\left(L_{k}\right)$, and further $\left|L_{k}\right| \leq C_{1}\left|J_{k}\right|$ for every such $k$. Moreover, by Lemma 8 there exists a constant $C_{2}>0$ such that $1-\left|\phi_{\nu}(z)\right|^{2} \leq C_{2}(1-|z|)$ for all $z \in S\left(L_{k}\right)$ when $k$ is sufficiently large, and it follows that

$$
\begin{aligned}
T_{k}(f) \leq & \frac{2 C_{1} C_{2}}{\left|L_{k}\right|} \int_{S\left(L_{k}\right)}\left|f_{\nu}^{\prime}(z)\right|^{2}\left(1-|z|^{2}\right) d A(z) \\
& +\frac{2 C_{1} C_{2}}{\left|L_{k}\right|} \int_{S\left(L_{k}\right)}\left|\frac{\phi_{\nu}^{\prime \prime}(z)}{\phi_{\nu}^{\prime}(z)}\right|^{2}\left(1-|z|^{2}\right) d A(z) \rightarrow 0, \quad k \rightarrow \infty,
\end{aligned}
$$

as $f_{\nu} \in \mathrm{VMOA}$ and $\log \phi_{\nu}^{\prime} \in \mathcal{D} \subset \mathrm{VMOA}$ by Lemma 8. Thus $f=\log g^{\prime} \in \mathrm{VMOA}$ as desired.

\section{Proof of Theorem 2}

4.1. Proof of $(\mathbf{1})$. If $g$ maps $\mathbb{D}$ conformally onto the inner domain of a Jordan curve, then the assertion in Part (1) is a direct consequence of $[20$, Corollary 2]. Let now $g \in \mathcal{H}(\mathbb{D})$ be locally univalent such that $\log g^{\prime} \in \mathcal{B}_{0}$ and $g^{\prime}(\zeta)=\lim _{r \rightarrow 1^{-}} g(r \zeta) \neq 0, \infty$ exists for $\zeta \in \mathbb{T}$. By Part (1) in Theorem $1, g$ is continuous on $\overline{\mathbb{D}}$ and thus $\mathcal{C}=g(\mathbb{T})$ is closed. Moreover, since $\log g^{\prime} \in \mathcal{B}_{0}$, the spherical derivative $\left(g^{\prime}\right)^{\#}(z)=\left|g^{\prime \prime}(z)\right| /\left(1+\left|g^{\prime}(z)\right|^{2}\right)$ of $g^{\prime}$ at $z$ satisfies

$$
\left(g^{\prime}\right)^{\#}(z)\left(1-|z|^{2}\right) \leq\left|\frac{g^{\prime \prime}(z)}{g^{\prime}(z)}\right|\left(1-|z|^{2}\right) \rightarrow 0, \quad|z| \rightarrow 1^{-} .
$$

Therefore $g^{\prime}$ is (strongly) normal in the sense of Lehto and Virtanen [17], and

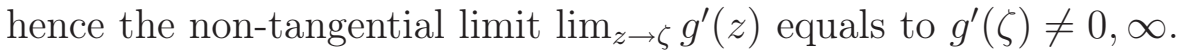

Define $\phi(t):=e^{i \zeta} \phi_{\delta, \rho}(t)$, where $\phi_{\delta, \rho}$ is as in Lemma 8 , and set $\phi(t)=z$ and $\phi(\tau)=\zeta$. Lemma 9 and Becker's univalence criterion [19, Theorem 1.11] show that $g \circ \phi$ is univalent and $\log (g \circ \phi)^{\prime} \in \mathcal{B}_{0}$ for suitably chosen $\delta$ and $\rho$. Moreover, by Lemma 8, the conformal map $\phi$ satisfies $\log \phi^{\prime} \in \mathcal{B}_{0}$ and $\lim _{r \rightarrow 1^{-}} \phi^{\prime}(r \tau)=\phi^{\prime}(\tau) \neq$ $0, \infty$. But now the non-tangential $\operatorname{limit}_{\lim _{z \rightarrow \zeta}} g^{\prime}(z)$ equals to $g^{\prime}(\zeta) \neq 0, \infty$, and hence $\lim _{r \rightarrow 1^{-}}(g \circ \phi)^{\prime}(r \tau) \neq 0, \infty$ exists. Since both $g \circ \phi$ and $\phi$ are conformal, it follows that

$$
\frac{g(z)-g(\zeta)}{z-\zeta}=\frac{g(\phi(t))-g(\phi(\tau))}{t-\tau} \frac{t-\tau}{\phi(t)-\phi(\tau)} \rightarrow \frac{(g \circ \phi)^{\prime}(\tau)}{\phi^{\prime}(\tau)}=g^{\prime}(\zeta)
$$

as $t \rightarrow \tau$, i.e., $z \rightarrow \zeta$. 
4.2. Proof of (2). In order to prove Part (2), we need the following auxiliary result which follows for instance from [20, Lemma 2].

Lemma A. Let $I \subset \mathbb{T}$ be an interval with midpoint $\xi$, and denote $t_{I}:=\xi(1-$ $|I| / 2)$. If $g$ is locally univalent and $\log g^{\prime} \in \mathrm{VMOA}$, then

$$
\frac{1}{|I|} \int_{I}\left|\frac{g^{\prime}(t)}{g^{\prime}\left(t_{I}\right)}-1\right||d t| \rightarrow 0, \quad|I| \rightarrow 0 .
$$

We now turn to prove (1.6). Without loss of generality, assume $s>\sigma$. Write $z=\psi(s)$ and $\zeta=\psi(\sigma)$, and denote $\mathbb{T}(\zeta, z):=\left\{e^{i t}: \arg \zeta \leq t \leq \arg z\right\}$. An application of Lemma A to $I=\left\{e^{i t}: 2 \arg \zeta-\arg z \leq t \leq \arg z\right\}$ yields

$$
\frac{1}{|z-\zeta|} \int_{\mathbb{T}(\zeta, z)}\left|\frac{g^{\prime}(t)}{g^{\prime}\left(t_{I}\right)}\right||d t| \rightarrow 1, \quad z \rightarrow \zeta
$$

Since $s \rightarrow \sigma$, as $z \rightarrow \zeta$, this and Part (1) give

$$
\frac{\omega(s)-\omega(\sigma)}{s-\sigma}=\frac{g(z)-g(\zeta)}{z-\zeta}\left(\frac{\left|g^{\prime}\left(t_{I}\right)\right|}{z-\zeta} \int_{\mathbb{T}(\zeta, z)}\left|\frac{g^{\prime}(t)}{g^{\prime}\left(t_{I}\right)}\right||d t|\right)^{-1} \rightarrow i \zeta \frac{g^{\prime}(\zeta)}{\left|g^{\prime}(\zeta)\right|},
$$

when $s \rightarrow \sigma$. Moreover,

$$
\arg \frac{\omega(s)-\omega(\sigma)}{s-\sigma} \rightarrow \arg i \zeta \frac{g^{\prime}(\zeta)}{\left|g^{\prime}(\zeta)\right|}=\frac{\pi}{2}+\arg \left(\zeta g^{\prime}(\zeta)\right)=\theta(\sigma), \quad s \rightarrow \sigma,
$$

and so $\omega^{\prime}(\sigma)=e^{i \theta(\sigma)}$.

4.3. Proof of (3). Recall that if $f \in \mathcal{D}$, then $f(\zeta)=\lim _{r \rightarrow 1^{-}} f(r \zeta) \neq \infty$ exists for all $\zeta \in \mathbb{T}$ outside of a possible exceptional set of zero logarithmic capacity by a classical result due to Beurling [4]. Hence, by Part (2), equation (1.6) is satisfied for all $\zeta \in \mathbb{T}$ outside of a set $A$ with $\operatorname{cap}(A)=0$. Hence it is satisfied for all $\sigma \in[0, l] \backslash E$, where $E=g(A)$. Now $g^{\prime} \in H^{2}$ by Theorem 1 , and therefore the inequality

$$
|g(z)-g(\zeta)|^{2} \leq\left(\int_{\mathbb{T}(\zeta, z)}\left|g^{\prime}(t)\right||d t|\right)^{2} \leq\left\|g^{\prime}\right\|_{H^{2}}^{2}|z-\zeta|
$$

shows that $g$ is $\frac{1}{2}$-Lipschitz, and hence

$$
\operatorname{cap}(E)=\operatorname{cap}(g(A)) \leq\left\|g^{\prime}\right\|_{H^{2}} \operatorname{cap}(A)=0
$$

by [24, Theorem 5.3.1].

\section{Proof of Theorem 4}

To prove (1.7) we may either first consider conformal maps and then apply the localization technique to deal with locally univalent functions, or prove the assertion directly for locally univalent functions. We will use the first option since the latter one requires a special care with notation as the preimages of points on self-intersecting curves are not necessarily unique. 
We begin with an auxiliary result which yields an upper estimate for the difference between the length of an arc and the distance between its end points in terms of a certain Poisson integral. To state the result in its full generality, we denote the Poisson kernel at $a$ by

$$
P_{a}(t):=\frac{1}{2 \pi} \frac{1-|a|^{2}}{|a-t|^{2}}, \quad a \in \mathbb{D}, \quad t \in \mathbb{T},
$$

and let $I_{a}:=\{z \in \mathbb{T}:|\arg z-\arg a|<\pi(1-|a|)\}$ be the interval on $\mathbb{T}$, induced by $a \in \mathbb{D}$, and of length $2 \pi(1-|a|)$.

Lemma 10. Let $h \in H^{1}$ and $a \in \mathbb{D}$. Then

$$
\frac{1}{\left|I_{a}\right|} \int_{I_{a}}|h(t)||d t|-\left|\frac{1}{\left|I_{a}\right|} \int_{I_{a}} h(t) d t\right| \leq 20\left(\int_{\mathbb{T}}|h(t)| P_{a}(t)|d t|-|h(a)|\right) .
$$

Proof. Let $a \in \mathbb{D}$. Then $P_{a}(t) \geq\left(20\left|I_{a}\right|\right)^{-1}$ for all $t \in I_{a}$. Therefore

$$
\begin{aligned}
|h(a)|-\left|\frac{1}{20\left|I_{a}\right|} \int_{I_{a}} h(t) d t\right| & \leq\left|\int_{\mathbb{T}}\left(h(t) P_{a}(t)-\frac{h(t) \chi_{I_{a}}(t)}{20\left|I_{a}\right|}\right) d t\right| \\
& \leq \int_{I_{a}}|h(t)|\left(P_{a}(t)-\frac{1}{20\left|I_{a}\right|}\right)|d t|+\int_{\mathbb{T} \backslash I_{a}}|h(t)| P_{a}(t)|d t| \\
& =\int_{\mathbb{T}}|h(t)| P_{a}(t)|d t|-\frac{1}{20\left|I_{a}\right|} \int_{I_{a}}|h(t)||d t|,
\end{aligned}
$$

and the assertion follows by rearranging terms.

To prove Theorem 4 , let first $g$ be a conformal map of $\mathbb{D}$ onto the inner domain of a Jordan curve $\mathcal{C}$ such that $\log g^{\prime} \in \mathcal{D}$. Then $\mathcal{C}$ is rectifiable by Part (3) in Theorem 1, and asymptotically smooth by [20, Theorem 2] as $\mathcal{D} \subset$ VMOA.

Let $w_{1}, w_{2} \in \mathcal{C}$, and let $\zeta_{1}, \zeta_{2} \in \mathbb{T}$ such that $g\left(\zeta_{i}\right)=w_{i}, i=1,2$. To each pair $w_{1}, w_{2}$ of distinct points on $\mathcal{C}$ there corresponds a unique $a=a\left(w_{1}, w_{2}\right) \in \mathbb{D}$ such that $I_{a}$ is the interval on $\mathbb{T}$ joining $\zeta_{1}$ and $\zeta_{2}$. With this notation Lemma 10 yields

$$
\begin{aligned}
I(g) & :=\int_{\mathcal{C}} \int_{\mathcal{C}} \frac{l\left(w_{1}, w_{2}\right)-\left|w_{1}-w_{2}\right|}{\left|w_{1}-w_{2}\right|^{3}}\left|d w_{1}\right|\left|d w_{2}\right| \\
& =\int_{\mathcal{C}} \int_{\mathcal{C}} \frac{\frac{1}{\left|I_{a}\right|} \int_{I_{a}}\left|g^{\prime}(t)\right||d t|-\left|\frac{1}{\left|I_{a}\right|} \int_{I_{a}} g^{\prime}(t) d t\right|}{\left|w_{1}-w_{2}\right|^{3}}\left|I_{a}\right|\left|d w_{1}\right|\left|d w_{2}\right| \\
& \leq 20 \int_{\mathcal{C}} \int_{\mathcal{C}} \frac{\left|I_{a}\right|}{\left|w_{1}-w_{2}\right|^{3}}\left(\int_{\mathbb{T}}\left|g^{\prime}(t)\right| P_{a}(t)|d t|-\left|g^{\prime}(a)\right|\right)\left|d w_{1}\right|\left|d w_{2}\right| .
\end{aligned}
$$

To estimate the Poisson integral above we use the relation

$$
\int_{\mathbb{T}}|h(t)|^{2} P_{a}(t)|d t|-|h(a)|^{2} \simeq \int_{\mathbb{D}}\left|h^{\prime}(z)\right|^{2}\left(1-\left|\varphi_{a}(z)\right|^{2}\right) d A(z), \quad h \in H^{2},
$$

where the constants of comparison are independent of $a$. This fact can be deduced by a standard application of Green's theorem, see, for example, [12, 
Chapter VI, Section 3]. Since $g$ is assumed to be univalent, we may apply (5.1) to $h=\left(g^{\prime}\right)^{\frac{1}{2}}$ to obtain

$$
\int_{\mathbb{T}}\left|g^{\prime}(t)\right| P_{a}(t)|d t|-\left|g^{\prime}(a)\right| \simeq \int_{\mathbb{D}} \frac{\left|g^{\prime \prime}(z)\right|^{2}}{\left|g^{\prime}(z)\right|}\left(1-\left|\varphi_{a}(z)\right|^{2}\right) d A(z) .
$$

It follows that there exists a constant $C_{1}>0$ such that

$$
I(g) \leq C_{1} \int_{\mathcal{C}} \int_{\mathcal{C}} \frac{\left|I_{a}\right|}{\left|w_{1}-w_{2}\right|^{3}}\left(\int_{\mathbb{D}} \frac{\left|g^{\prime \prime}(z)\right|^{2}}{\left|g^{\prime}(z)\right|}\left(1-\left|\varphi_{a}(z)\right|^{2}\right) d A(z)\right)\left|d w_{1}\right|\left|d w_{2}\right| .
$$

To estimate the integral over $\mathbb{D}$, we split $\mathbb{D}$ into pieces by using Carleson boxes. To do this, for $a \in \mathbb{D}$, choose $N=N(a) \in \mathbb{N}$ such that $2^{N}(1-|a|)<1$ and $2^{N+1}(1-|a|) \geq 1$. Set $S_{-1}=\emptyset$,

$$
S_{k}(a):=\left\{z \in \mathbb{D}: 1-|z|<2^{k}(1-|a|),|\arg z-\arg a|<2^{k} \pi(1-|a|)\right\}
$$

for $k=0, \ldots, N$ and $S_{N+1}(a)=\mathbb{D} \backslash S_{N}(a)$. Then there exists a constant $C_{2}>0$ such that

$$
C_{2}^{-1} 2^{k}(1-|a|) \leq|1-\bar{a} z| \leq C_{2} 2^{k}(1-|a|)
$$

for all $z \in S_{k}(a) \backslash S_{k-1}(a)$ and $k=0, \ldots, N+1$. It follows that

$$
\int_{\mathbb{D}} \frac{\left|g^{\prime \prime}(z)\right|^{2}}{\left|g^{\prime}(z)\right|}\left(1-\left|\varphi_{a}(z)\right|^{2}\right) d A(z) \leq \sum_{k=0}^{N+1} \frac{2 C_{2}^{2}}{4^{k}\left|I_{a}\right|} \int_{S_{k}(a)} \frac{\left|g^{\prime \prime}(z)\right|^{2}}{\left|g^{\prime}(z)\right|}\left(1-|z|^{2}\right) d A(z) .
$$

This together with Fubini's theorem give

$$
\begin{aligned}
I(g) & \leq C_{3} \int_{\mathcal{C}} \int_{\mathcal{C}} \frac{1}{\left|w_{1}-w_{2}\right|^{3}}\left(\sum_{k=0}^{N+1} \frac{1}{4^{k}} \int_{S_{k}(a)} \frac{\left|g^{\prime \prime}(z)\right|^{2}}{\left|g^{\prime}(z)\right|}\left(1-|z|^{2}\right) d A(z)\right)\left|d w_{1}\right|\left|d w_{2}\right| \\
& =C_{3} \sum_{k=0}^{N+1} \frac{1}{4^{k}} \int_{\mathcal{C}} \int_{\mathcal{C}} \frac{1}{\left|w_{1}-w_{2}\right|^{3}}\left(\int_{S_{k}(a)} \frac{\left|g^{\prime \prime}(z)\right|^{2}}{\left|g^{\prime}(z)\right|}\left(1-|z|^{2}\right) d A(z)\right)\left|d w_{1}\right|\left|d w_{2}\right| \\
& =C_{3} \int_{\mathbb{D}} \frac{\left|g^{\prime \prime}(z)\right|^{2}}{\left|g^{\prime}(z)\right|}\left(1-|z|^{2}\right) \sum_{k=0}^{N+1} \frac{1}{4^{k}}\left(\int_{E_{1, k}(z)} \int_{E_{2, k}(z)} \frac{\left|d w_{1}\right|\left|d w_{2}\right|}{\left|w_{1}-w_{2}\right|^{3}}\right) d A(z),
\end{aligned}
$$

where $C_{3}=2 C_{1} C_{2}^{2}$ and the sets $E_{1, k}(z)$ and $E_{2, k}(z)$ consist of those $w_{1}$ and $w_{2}$ on $\mathcal{C}$ for which $S_{k}(a)$ contains the point $z$. To estimate the double integral involving these sets, the following lemma is used.

Lemma 11. Let $t$ and $\tau$ such that $0<\tau-t<2 \pi$, and define

$$
S\left(e^{i t}, e^{i \tau}\right):=\left\{z=r e^{i \phi} \in \mathbb{D}: t<\phi<\tau, r>1-(\tau-t) / 2 \pi\right\} .
$$

Let $z \in \mathbb{D}$ and let $F_{1}(z)$ and $F_{2}(z)$ be the sets of points $\zeta_{1}$ and $\zeta_{2}$ on $\mathbb{T}$ for which $S\left(\zeta_{1}, \zeta_{2}\right)$ contains $z$. Then

$$
A(z):=\int_{F_{1}(z)} \int_{F_{2}(z)} \frac{\left|d \zeta_{1}\right|\left|d \zeta_{2}\right|}{\left|\zeta_{1}-\zeta_{2}\right|^{p}} \simeq\left\{\begin{array}{cc}
(1-|z|)^{2-p}, & p>2 \\
\log \frac{1}{1-|z|}, & p=2 \\
1, & p<2 .
\end{array}\right.
$$


Proof. The quantity $A(z)$ depends only on $|z|$, so we may consider $|z|$ instead of $z$. Then the definition of $S\left(\zeta_{1}, \zeta_{2}\right)$ yields

$$
\begin{aligned}
A(|z|) & =\int_{0}^{2 \pi(1-|z|)} \int_{\theta-2 \pi}^{\theta-2 \pi(1-|z|)} \frac{d \phi d \theta}{\mid e^{i \theta}-e^{i \phi \mid p}}+\int_{2 \pi(1-|z|)}^{2 \pi} \int_{\theta-2 \pi}^{0} \frac{d \phi d \theta}{\mid e^{i \theta}-e^{i \phi \mid p}} \\
& =\int_{0}^{2 \pi(1-|z|)} \int_{-2 \pi}^{-2 \pi(1-|z|)} \frac{d t d \theta}{(2(1-\cos t))^{\frac{p}{2}}}+\int_{2 \pi(1-|z|)}^{2 \pi} \int_{-2 \pi}^{-\theta} \frac{d t d \theta}{(2(1-\cos t))^{\frac{p}{2}}} \\
& =2 \pi(1-|z|) \int_{2 \pi(1-|z|)}^{2 \pi} \frac{d t}{(2(1-\cos t))^{\frac{p}{2}}}+\int_{2 \pi(1-|z|)}^{2 \pi} \int_{\theta}^{2 \pi} \frac{d t d \theta}{(2(1-\cos t))^{\frac{p}{2}}} \\
& =: A_{1}(|z|)+A_{2}(|z|) .
\end{aligned}
$$

By using the Taylor series expansion of cost, we obtain

$$
A_{1}(|z|) \simeq\left\{\begin{aligned}
\frac{1}{(1-|z|)^{p-2}}-(1-|z|), & \text { if } p>1 \\
o(1), \quad|z| \rightarrow 1^{-}, & \text {if } p \leq 1
\end{aligned}\right.
$$

and

$$
A_{2}(|z|) \simeq\left\{\begin{array}{cl}
\frac{1}{(1-|z|)^{p-2}}-\frac{1}{p-2}-|z|, & \text { if } p>2 \\
\log \frac{1}{1-|z|}-|z|, & \text { if } p=2 \\
O(1), \quad|z| \rightarrow 1^{-}, & \text {if } p<2
\end{array}\right.
$$

The assertions follow by combining these asymptotic relations.

We turn back to the proof of Theorem 4. It is clear that

$$
t \in S_{k}(|a|) \quad \Leftrightarrow \quad\left(1-(1-|t|) / 2^{k}\right) \exp \left(i \frac{\arg t}{2^{k}}\right) \in S_{0}(|a|), \quad k=1, \ldots, N,
$$

and hence Lemma 11 yields

$$
\int_{F_{1, k}(z)} \int_{F_{2, k}(z)} \frac{\left|d \zeta_{1}\right|\left|d \zeta_{2}\right|}{\left|\zeta_{1}-\zeta_{2}\right|^{3}} \simeq \frac{2^{k}}{1-|z|}=\frac{2^{k}}{\operatorname{dist}(z, \mathbb{T})}
$$

where $F_{1, k}(z)$ and $F_{2, k}(z)$ are the sets of points $\zeta_{1}$ and $\zeta_{2}$ on $\mathbb{T}$ for which $S_{k}(a)$ contains $z \in \mathbb{D}$. Since $\mathcal{C}$ is asymptotically smooth, and thus a quasi circle, the conformal map $g$ has a quasi-conformal extension over the whole plane. Therefore we may apply the circular distortion theorem [16, Theorem 2.4] to find a constant $C_{4}>0$ such that

$$
\frac{1}{C_{4}} \frac{2^{k}}{\operatorname{dist}(g(z), \mathcal{C})} \leq \int_{E_{1, k}(z)} \int_{E_{2, k}(z)} \frac{\left|d w_{1}\right|\left|d w_{2}\right|}{\left|w_{1}-w_{2}\right|^{3}} \leq C_{4} \frac{2^{k}}{\operatorname{dist}(g(z), \mathcal{C})}
$$

for all $z \in \mathbb{D}$. This along with the inequality $\operatorname{dist}(g(z), \mathcal{C}) \geq\left|g^{\prime}(z)\right|\left(1-|z|^{2}\right) / 4$ (see [19, Corollary 1.4], for instance) yields

$$
I(g) \leq 4 C_{3} C_{4} \int_{\mathbb{D}}\left|\frac{g^{\prime \prime}(z)}{g^{\prime}(z)}\right|^{2} d A(z) \sum_{k=0}^{\infty} \frac{1}{2^{k}} \leq 4 C_{3} C_{4}\left\|\log g^{\prime}\right\|_{\mathcal{D}}^{2}<\infty .
$$


This concludes the proof of Theorem 4 in the case when $g$ maps $\mathbb{D}$ conformally onto the inner domain of a Jordan curve $\mathcal{C}$.

Let now $g$ be locally univalent such that $\log g^{\prime} \in \mathcal{D}$. Then $g(\mathbb{T})$ must be closed by Theorem 1, and hence asymptotically smooth by Theorem 3 . Further, by Lemma 9 there exist $\rho \in(0,1)$ and $\delta_{0} \in(0,(1-\rho) / 2)$ such that $\tilde{g}_{\delta, \rho}=g \circ \phi_{\delta, \rho}$ satifies

$$
\left\|\log \tilde{g}_{\delta, \rho}^{\prime}\right\|_{\mathcal{B}}<1 \quad \text { and } \quad \log \tilde{g}_{\delta, \rho}^{\prime} \in \mathcal{D}
$$

for all $\delta \in\left(0, \delta_{0}\right)$. Becker's univalence criterion [19, Theorem 1.11] shows that $\tilde{g}_{\delta, \rho}$ is univalent, and hence $\tilde{g}_{\delta, \rho}(\mathbb{T})$ is a Jordan curve. Therefore the first part of the proof shows that $(1.8)$, with $\tilde{g}_{\delta, \rho}(\mathbb{T})$ in place of $\mathcal{C}$, is satisfied.

Let $I$ be an arc on $\mathbb{T}$ such that $|I|<2 \delta_{0}$, and let $\zeta=e^{i t} \in \mathbb{T}$ be the midpoint of $I$. Set $\phi_{I}:=e^{i t} \phi_{|I|, \rho}(z)$ and define $g_{I}:=g \circ \phi_{I}$. Since the Diriclet space $\mathcal{D}$ is invariant under rotations, the Jordan curve $g_{I}(\mathbb{T})$ must have the same properties as $\tilde{g}_{|I|, \rho}(\mathbb{T})$. It follows that

$$
\int_{g(I)} \int_{g(I)} \frac{l\left(w_{1}, w_{2}\right)-\left|w_{1}-w_{2}\right|}{\left|w_{1}-w_{2}\right|^{3}}\left|d w_{1}\right|\left|d w_{2}\right|<\infty
$$

for any interval $I$ on $\mathbb{T}$ such that $|I|<2 \delta_{0}$. This clearly implies that (1.7) must be satisfied.

\section{Proof of Theorem 5}

Theorem 5 is based on the following known result which proof is given for the convenience of the reader. The work of Douglas [10] is probably the most original reference regarding to the identity (6.1).

Theorem B. If $f \in H^{2}$, then

$$
\begin{aligned}
\|f\|_{\mathcal{D}}^{2}-|f(0)|^{2} & =\frac{1}{4 \pi^{2}} \int_{\mathbb{T}} \int_{\mathbb{T}}\left|\frac{f(z)-f(\zeta)}{z-\zeta}\right|^{2}|d z||d \zeta| \\
& =\frac{1}{2 \pi^{2}} \int_{0}^{2 \pi} \int_{0}^{2 \pi}\left(\frac{\Re\left(f\left(e^{i t}\right)-f\left(e^{i \tau}\right)\right)}{2 \sin ((t-\tau) / 2)}\right)^{2} d t d \tau \\
& =\frac{1}{2 \pi^{2}} \int_{0}^{2 \pi} \int_{0}^{2 \pi}\left(\frac{\Im\left(f\left(e^{i t}\right)-f\left(e^{i \tau}\right)\right)}{2 \sin ((t-\tau) / 2)}\right)^{2} d t d \tau .
\end{aligned}
$$

Proof. To prove (6.1), write $f(z)=\sum_{n=0}^{\infty} a_{n} z^{n}$ and set $\zeta=z e^{i \tau}$. Then the identity

$$
1-\cos \tau=2 \sin ^{2}(\tau / 2)
$$

shows that the integral in (6.1) equals to

$$
I_{1}(f):=\frac{1}{2 \pi} \int_{0}^{2 \pi}\left(\frac{1}{2 \pi} \int_{\mathbb{T}}\left|f(z)-f\left(z e^{i \tau}\right)\right|^{2}|d z|\right) \frac{d \tau}{4 \sin ^{2}(\tau / 2)},
$$


where

$$
f(z)-f\left(z e^{i \tau}\right)=\sum_{n=1}^{\infty} a_{n}\left(1-e^{i n \tau}\right) z^{n} .
$$

Parseval's formula, (6.4) and the identity

$$
\int_{0}^{2 \pi} \frac{\sin ^{2}(n \tau / 2)}{\sin ^{2}(\tau / 2)} d \tau=\pi n
$$

yield

$$
I_{1}(f)=\frac{1}{2 \pi} \int_{0}^{2 \pi} \sum_{n=1}^{\infty}\left|a_{n}\right|^{2} \frac{\sin ^{2}(n \tau / 2)}{\sin ^{2}(\tau / 2)} d \tau=\sum_{n=1}^{\infty} n\left|a_{n}\right|^{2}=\|f\|_{\mathcal{D}}^{2}-|f(0)|^{2} .
$$

To prove (6.2) and (6.3), note first that for $h \in H^{2}$ with $h(0)=0$ we have

$$
\frac{1}{2 \pi} \int_{\mathbb{T}}|h(z)|^{2}|d z|=\frac{1}{\pi} \int_{\mathbb{T}}(\Re h(z))^{2}|d z|=\frac{1}{\pi} \int_{\mathbb{T}}(\Im h(z))^{2}|d z|
$$

by [12, p. 104; Theorem 1.5]. For $\zeta \in \mathbb{T}$, set

$$
h_{\zeta}(z):=z \zeta \frac{f\left(z^{2}\right)-f\left(\zeta^{2}\right)}{z^{2}-\zeta^{2}}, \quad z \in \mathbb{D} .
$$

Then $h_{\zeta}(0)=0$ and, since (6.1) is proved, we may assume $h_{\zeta} \in H^{2}$. Setting $z=e^{i t / 2}$ and $\zeta=e^{i \tau / 2}$ we have

$$
\left|h_{\zeta}(z)\right|=\left|\frac{f\left(e^{i t}\right)-f\left(e^{i \tau}\right)}{e^{i t}-e^{i \tau}}\right| .
$$

A direct calculation based on (6.4) shows that $z \zeta /\left(z^{2}-\zeta^{2}\right)=(2 i \sin ((t-\tau) / 2))^{-1}$, and hence

$$
\Re h_{\zeta}(z)=\frac{\Im\left(f\left(e^{i t}\right)-f\left(e^{i \tau}\right)\right)}{2 \sin ((t-\tau) / 2)} \quad \text { and } \quad \Im h_{\zeta}(z)=-\frac{\Re\left(f\left(e^{i t}\right)-f\left(e^{i \tau}\right)\right)}{2 \sin ((t-\tau) / 2)} .
$$

The identities (6.2) and (6.3) now follow at once by integrating (6.5) with respect to $\zeta$.

To prove Theorem 5 , set $f=\log g^{\prime}$ in (6.3) to obtain

$$
\left\|\log g^{\prime}\right\|_{\mathcal{D}}^{2}=\left|\log g^{\prime}(0)\right|^{2}+\frac{1}{2 \pi^{2}} \int_{0}^{2 \pi} \int_{0}^{2 \pi}\left(\frac{\arg g^{\prime}\left(e^{i t}\right)-\arg g^{\prime}\left(e^{i \tau}\right)}{2 \sin ((t-\tau) / 2)}\right)^{2} d t d \tau .
$$

Since

$$
\frac{\left|e^{i t}-e^{i \tau}\right|}{\pi} \leq \frac{|t-\tau|}{\pi} \leq\left|\sin \frac{t-\tau}{2}\right| \leq \frac{|t-\tau|}{2} \leq \frac{\pi}{4}\left|e^{i t}-e^{i \tau}\right|,
$$

whenever $|t-\tau| \leq \frac{\pi}{2}$, it follows that $\log g^{\prime} \in \mathcal{D}$ if and only if $\log g^{\prime} \in H^{2}$ and (1.9) is satisfied.

Consider now the arc length parametrization $\omega$ for which

$$
\omega(s)=g(\psi(s)), \quad \omega^{\prime}(s)=g^{\prime}(\psi(s)) \psi^{\prime}(s)
$$


almost everywhere on $[0, l(g(\mathbb{T}))]$. It is known that

$$
|\psi(s)|=1, \quad \Re \frac{\psi^{\prime}(s)}{\psi(s)}=0 \quad \text { and } \quad \arg \psi^{\prime}(\mathrm{s})=\arg \psi(\mathrm{s})+\frac{\pi}{2},
$$

and therefore, for $z=\psi(s)$ and $\zeta=\psi(\sigma)$, we have

$$
\arg g^{\prime}(z)-\arg g^{\prime}(\zeta)=\arg \omega^{\prime}(s)-\arg \omega^{\prime}(\sigma)-(\arg \psi(s)-\arg \psi(\sigma)) .
$$

It follows that

$$
\frac{\arg g^{\prime}(z)-\arg g^{\prime}(\zeta)}{z-\zeta}=\frac{\arg \omega^{\prime}(s)-\arg \omega^{\prime}(\sigma)}{z-\zeta}-\frac{\arg z-\arg \zeta}{z-\zeta} .
$$

Since the last quotient is uniformly bounded for $z, \zeta \in \mathbb{D}$ we deduce that the conditions (1.9) and (1.10) are equivalent.

\section{Proof of Corollary 6}

Note first that the assumption (1.11) yields $\left|\Re \log g^{\prime}(z)\right| \leq \log \frac{1}{c}$ and therefore $\log g^{\prime} \in H^{2}$. Setting $z=\psi(s)$ and $\zeta=\psi(\sigma)$, the integral in (1.10) becomes

$$
\int_{0}^{l} \int_{0}^{l}\left(\frac{\arg \omega^{\prime}(s)-\arg \omega^{\prime}(\sigma)}{s-\sigma}\right)^{2} \frac{\left|\psi^{\prime}(s) \psi^{\prime}(\sigma)\right||s-\sigma|^{2}}{|\psi(s)-\psi(\sigma)|^{2}} d s d \sigma .
$$

Now $\left|\psi^{\prime}(s)\right|=\left|g^{\prime}(z)\right|^{-1}$ by (6.6), and hence

$$
\frac{\left|\psi^{\prime}(s) \psi^{\prime}(\sigma)\right||s-\sigma|^{2}}{|\psi(s)-\psi(\sigma)|^{2}}=\frac{1}{\left|g^{\prime}(z) g^{\prime}(\zeta)\right|}\left(\int_{\mathbb{T}(z, \zeta)}\left|g^{\prime}(t)\right||d t|\right)^{2} \frac{1}{|z-\zeta|^{2}} .
$$

Therefore (1.11) yields

$$
c^{4} \leq \frac{\left|\psi^{\prime}(s) \psi^{\prime}(\sigma)\right||s-\sigma|^{2}}{|\psi(s)-\psi(\sigma)|^{2}} \leq \frac{\pi^{2}}{c^{4}}
$$

and thus Corollary 6 follows by (7.1).

\section{Proof of Theorem 7}

To prove (1), assume $\mathcal{C} \in \Lambda^{1, \alpha}$ for some $\alpha \in\left(\frac{1}{2}, 1\right)$. The substitutions $z=e^{i t}$ and $w=e^{i(t+\tau)}$ in (1.9) yields

$$
I_{2}(g):=\int_{0}^{2 \pi}\left(\int_{0}^{2 \pi}\left(\arg g^{\prime}\left(e^{i t}\right)-\arg g^{\prime}\left(e^{i(t+\tau)}\right)\right)^{2} d t\right) \frac{d \tau}{\left|1-e^{i \tau}\right|^{2}} .
$$

By the assumption $\mathcal{C} \in \Lambda^{1, \alpha}$, (1.14) and (6.4) we obtain

$$
I_{2}(g) \leq 2 \pi C_{2}^{2} \int_{0}^{2 \pi}\left|1-e^{i \tau}\right|^{2(\alpha-1)} d \tau \leq 2 \pi C_{2}^{2} \int_{0}^{2 \pi}\left(\sin \frac{\tau}{2}\right)^{2(\alpha-1)} d \tau .
$$

The last integral converges since $\alpha>1 / 2$, and hence $\log g^{\prime} \in \mathcal{D}$ by Theorem 5 . 
To show (2), consider the lacunary series $f(z):=\sum_{n=0}^{\infty} 2^{-n} z^{4^{n}}, z \in \mathbb{D}$. By using the standard method for estimating such series we obtain

$$
\frac{r f^{\prime}(r)}{1-r}=\frac{1}{1-r} \sum_{n=0}^{\infty} 2^{n} r^{4^{n}}=\sum_{k=1}^{\infty}\left(\sum_{4^{n} \leq k} 2^{n}\right) r^{k} \leq \sum_{k=1}^{\infty} 2 \sqrt{k} r^{k} \leq C_{3} r(1-r)^{-3 / 2}
$$

for all $0<r<1$. Therefore

$$
\left|f^{\prime}(z)\right| \leq C_{3}\left(1-|z|^{2}\right)^{-1 / 2}, \quad z \in \mathbb{D},
$$

from which [11, Theorem 5.1] yields

$$
|f(z)-f(w)| \leq C_{4}|z-w|^{\frac{1}{2}}, \quad z, w \in \overline{\mathbb{D}} .
$$

Define $g$ by $\log g^{\prime}=\lambda f$, where $\lambda>0$. Since $f \in \mathcal{B}$ by (8.1), we may choose $\lambda$ such that $\left\|\log g^{\prime}\right\|_{\mathcal{B}}<1$ and then $g$ is univalent by Becker's univalence criterion [19, Theorem 1.11]. Moreover,

$$
\left|\arg g^{\prime}(z)-\arg g^{\prime}(w)\right| \leq\left|\log g^{\prime}(z)-\log g^{\prime}(w)\right|=\lambda|f(z)-f(w)|,
$$

and so $\mathcal{C} \in \Lambda^{1, \frac{1}{2}}$ by $(8.2)$. But $\log g^{\prime}=\lambda f \notin \mathcal{D}$ since $\sum_{n=0}^{\infty} 4^{n}\left(2^{-n}\right)^{2}$ diverges.

\section{REFERENCES}

[1] K. Astala and M. Zinsmeister, Teichmüller spaces and BMOA, Math. Ann. 289(4) (1991), 613-625.

[2] J. Becker, Löwnersche Differentialgleichung und quasikonform fortsetzbare schlichte Funktionen, J. Reine Angew. Math. 255 (1972), 23-43.

[3] J. Becker and Ch. Pommerenke, Über die quasikonforme Fortsetzung schlichter Funktionen, Math. Z. 161 (1978), 69-80.

[4] A. Beurling, Ensembles exceptionnels, Acta Math. 72 (1940), 1-13.

[5] C. J. Bishop and P. W. Jones, Harmonic measure, $L^{2}$ estimates and the Schwarzian derivative, J. Anal. Math. 62 (1994), 77-113.

[6] L. Carleson, Interpolations by bounded functions and the corona problem, Ann. of Math. 76 (1962), 547-559.

[7] L. Carleson, Selected problems on exceptional sets, D. Van Nostrand Company, Inc. 1966.

[8] S.-Y. Chang and D. Marshall, On a sharp inequality concerning the Dirichlet integral, Amer. J. Math. 107(5) (1985), 1015-1033.

[9] J. Cima and G. Schober, Analytic functions with bounded mean oscillation and logarithms of $H^{p}$ functions, Math. Z. 151(3) (1976), 295-300.

[10] J. Douglas, Solution of the problem of Plateau, Trans. Amer. Math. Soc. 33 (1931), 263-321.

[11] P. Duren, Theory of $H^{p}$ spaces, Academic Press, New York-London 1970.

[12] J. B. Garnett, Bounded analytic functions, Revised first edition. Graduate Texts in Mathematics, 236. Springer, New York, 2007.

[13] J. B. Garnett and D. E. Marshall, Harmonic measure, New Mathematical Monographs, 2. Cambridge University Press, Cambridge, 2005.

[14] P. W. Jones, Personal communication.

[15] P. Koosis, The logarithmic integral II, Cambridge University Press, 1992.

[16] O. Lehto, Univalent functions and Teichmüller spaces, Graduate Texts in Mathematics, 109. Springer-Verlag, New York, 1987. 
[17] O. Lehto and K. I. Virtanen, Boundary behaviour and normal meromorphic functions, Acta Math. 97 (1957), 47-65.

[18] F. Pérez-González and J. Rättyä, Dirichlet and VMOA domains via Schwarzian derivative, J. Math. Anal. Appl. 359 (2009), 543-546.

[19] Ch. Pommerenke, Boundary behaviour of conformal maps, Springer-Verlag, Berlin, 1992.

[20] Ch. Pommerenke, On univalent functions, Bloch functions and VMOA, Math. Ann. 236(3) (1978), 199-208.

[21] Ch. Pommerenke, Schlichte Funktionen und analytische Funktionen von beschränkter mittlerer Oszillation (German), Comment. Math. Helv. 52(4) (1977), 591-602.

[22] Ch. Pommerenke, Univalent Functions, Vandenhoeck und Ruprecht, Göttingen, 1975.

[23] Ch. Pommerenke and S. E. Warschawski, On the quantitative boundary behavior of conformal maps, Comment. Math. Helv. 57(1) (1982), 107--129.

[24] T. Ransford, Potential theory in the complex plane, London Mathematical Society Student Texts, 28, Cambridge University Press, Cambridge, 1995.

[25] B. Rodin and S. E. Warschawski, Conformal mapping and locally asymptotically conformal curves, Proc. London Math. Soc. (3), 49 (1984), 255-273.

[26] S. E. Warschawski, On mean convergence of conformal mapping, Arch. Math. 6 (1955), $102-114$.

[27] A. Zygmund, Trigonometric Series, Cambridge University Press, London, 1959.

Eva A. Gallardo-Gutiérrez

DEPARTAMENTO DE MATEMÁtiCAS

UNIVERSIDAD DE ZARAGOZA E IUMA

Plaza San Francisco S/N

50009 Zaragoza, Spain

E-mail address: eva@unizar.es

María J. GonzÁlez

Departamento de Matemáticas

UNIVERSIDAD DE CÁDIZ

Apartado 40, 11510 Puerto Real (CÁdiz), Spain

E-mail address: majose.gonzalez@uca.es

Fernando Pérez-González

Departamento de Análisis Matemático

UNIVERSIDAD DE LA LAGUNA

38271, LA LAGUNA, SPAIN

E-mail address: fernando.perez.gonzalez@ull.es

Christian Pommerenke

INSTITUT FÜR MATHEMATIK

MA 8-2, TECHNISCHE UNIVERSITÄT

D-10623 Berlin, Germany

E-mail address: pommeren@math.tu-berlin.de

JOUNI RÄTTYÄ

UNIVERSITY OF JOENSUU

Department of Physics And Mathematics

PO Box 111, 80101 Joensuu, Finland

E-mail address: jouni.rattya@joensuu.fi 